\title{
Ground State(s) of the Spin-Boson Hamiltonian
}

\author{
Herbert Spohn*
}

Department of Physics, University of California, Santa Barbara, CA 93106, USA

\begin{abstract}
We establish that the finite temperature KMS states of the spin-boson hamiltonian have a limit as the temperature drops to zero. Using recent advances on the one-dimensional Ising model with long range, $1 / r^{2}$, interactions, we prove qualitative properties of the ground state(s) in the ohmic case. We show (i) the asymptotics of the critical coupling as the bare energy gap goes to zero and to infinity, (ii) a jump in the order parameter, and (iii) that the number of bosons is finite below and infinite at and above the critical coupling strength.
\end{abstract}

\section{Introduction}

The spin-boson Hamiltonian models a "spin" coupled to a Bose field and is given by

$$
H=-\varepsilon \sigma_{x}+\int d k \omega(k) a^{+}(k) a(k)+\sqrt{\alpha / 2} \sigma_{z} \int d k \lambda(k)\left(a^{+}(k)+a(k)\right)-h \sigma_{z}
$$

Here $\sigma_{x}, \sigma_{z}$ are the Pauli spin matrices. $a(k), a^{+}(k)$ is a scalar Bose field with commutation relation $\left[a(k), a^{+}\left(k^{\prime}\right)\right]=\delta\left(k-k^{\prime}\right)$. The Bose field is over $\mathbf{R}^{d}$, the $d$-dimensional Euclidean space. $\omega(k)$ is the dispersion relation of the field, $\omega(k) \geqq 0$. $\lambda(k)=\lambda(k)^{*}$ are the couplings.

The spin-boson system is a prototype for the interaction of a localized degree of freedom with a field. No wonder the spin-boson Hamiltonian has a rich history. The reader is referred to [1] for an excellent and up-to-date review.

We consider here only the case characteristic of most physical applications, the so-called ohmic case: the frequency distribution is linear for small $\omega$, i.e.

Equivalently

$$
\int d k \lambda(k)^{2} \delta(\omega(k)-\omega) \simeq \omega .
$$

$$
W(t)=\int d k \lambda(k)^{2} e^{-\omega(k)|t|} \simeq \frac{1}{t^{2}}
$$

^ Permanent address: Theoretische Physik, Universität München, Theresienstr. 37, D-8000 München

2, Federal Republic of Germany 
for large $t$. More precisely, the coupling constant $\alpha$ is normalized in such a way that

$$
\lim _{t \rightarrow \infty} t^{2} W(t)=1
$$

To complete our assumptions we require that

$$
\int d k \lambda(k)^{2}<\infty
$$

which amounts to $W(t)$ being bounded. More singular cases could be dealt with if needed. We regard $\lambda$ and $\omega$, hence $W$, as given. $h=0$, with the proviso $h \rightarrow 0_{ \pm}$ in case of symmetry breaking. We investigate the structure of the ground state(s) of $H$ in dependence on the coupling strength $\alpha$ and on the (bare) energy splitting $\varepsilon, \alpha \geqq 0, \varepsilon \geqq 0$.

In [2] we studied the ground state of the spin-boson Hamiltonian exploiting the connection to the one-dimensional, continuum Ising model with long range interactions. In particular we showed that the "flip" symmetry is broken for sufficiently large $\alpha$.

In the recent two years the rigorous Ising technology for one-dimensional lattice systems with ferromagnetic long range interactions has staged spectacular progress. The purpose of this note is to indicate how the proofs of Aizenman and Newman [3] and of Aizenman, Chayes, Chayes, and Newman [4] extend to the spin-boson Hamiltonian. In particular we show

- bounds on the critical coupling strength $\alpha_{c}(\varepsilon)$ which imply $1 \leqq \alpha_{c}(\varepsilon) \leqq 2$ as $\varepsilon \rightarrow 0$ and $\alpha_{c}(\varepsilon)=\left(\int_{0}^{\infty} d t W(t)\right)^{-1} \varepsilon+0(\varepsilon)$ as $\varepsilon \rightarrow \infty\left(\alpha_{c}(\varepsilon)\right.$ is increasing in $\left.\varepsilon\right)$,

- jump in the order parameter across $\alpha_{c}(\varepsilon)$,

- the asymptotic decay of the average boson density in space for all $\alpha$.

The theorems are stated in Sect. 3. Their proofs are given in Sects. 5 to 8 .

We use the opportunity to establish that the finite temperature KMS states of the spin-boson Hamiltonian without Bose-Einstein condensate converge to the ground states studied here in the limit of zero temperature, cf. Sects. 2 and 4.

\section{Ground States as Temperature Zero Limit of Thermal States}

If

$$
\int d k \lambda(k)^{2}<\infty, \quad \int d k \lambda(k)^{2} / \omega(k)<\infty,
$$

then $H$ is self-adjoint on $\mathbf{C}^{2} \otimes \mathscr{F}_{s}$, where $\mathscr{F}_{s}$ denotes the standard Boson Fock space over $\mathbf{R}^{d}$ of symmetric wave functions. A domain of essential self-adjointness is the domain of $-\varepsilon \sigma_{x}+\int d k \omega(k) a^{+}(k) a(k)$. $H$ is bounded from below as

$$
\langle\psi \mid H \psi\rangle \geqq-\left(\varepsilon+|h|+\int d k \lambda(k)^{2} / \omega(k)\right)\langle\psi \mid \psi\rangle .
$$

Thus, a ground state is a vector $\psi \in \mathbf{C}^{2} \otimes \mathscr{F}_{s}$ which minimizes

$$
\langle\psi \mid H \psi\rangle,\langle\psi \mid \psi\rangle=1
$$

This standard definition of a ground state is not adequate for our purposes, 
because of the possibly large number of infrared bosons. To see why we consider the particular case $\varepsilon=0$, i.e.

$$
H_{0}=\int d k \omega(k) a^{+}(k) a(k)+\sqrt{\alpha / 2} \sigma_{z} \int d k \lambda(k)\left(a^{+}(k)+a(k)\right)-h \sigma_{z} .
$$

For $h=0 H_{0}$ has two ground states related by the flip symmetry, $\sigma_{z} \rightarrow-\sigma_{z}$, $a(k) \rightarrow-a(k) . h$ breaks this symmetry. In the ground state the number $n(\Lambda)$, of bosons in the region $\Lambda$ of momentum space has the Poisson distribution

$$
\operatorname{Prob}(\{n(\Lambda)=n\})=\frac{1}{n !}\left(\frac{\alpha}{2} \int_{\Lambda} d k \lambda(k)^{2} / \omega(k)^{2}\right)^{n} \exp \left[-\frac{\alpha}{2} \int_{\Lambda} d k \lambda(k)^{2} / \omega(k)^{2}\right],
$$

where $\Lambda$ is such that $\int_{\Lambda} d k \lambda(k)^{2} / \omega(k)^{2}<\infty$ and $\Lambda \subset\left\{k \in \mathbf{R}^{d} \mid \omega(k) \neq 0\right\}$. For disjoint regions the $n(\Lambda)$ 's are independent. Thus if $\int d k \lambda(k)^{2} / \omega(k)^{2}=\infty$, then the number of bosons is infinite and the ground state does not lie in Fock space. On the other hand the ground state energy equals

$$
-\left(\frac{\alpha}{2} \int d k \lambda(k)^{2} / \omega(k)+|h|\right)
$$

which is finite by (2.1). Note that $\int d k(\lambda / \omega)^{2}=\infty$ for (1.2), (1.3).

In view of this situation we define "ground state" as the temperature zero limit of a thermal state. One could adopt other definitions, cf. the discussion below. A thermal state has an infinite number of bosons. Therefore, the state has to be defined on a suitable algebra of quasi-local observables. This construction has been carried through in [5]. The algebra of observables is $M_{2} \otimes \mathscr{A} \cdot M_{2}$ are the $2 \times 2$ complex matrices. Let $\tilde{\mathscr{H}}$ be the Hilbert space with inner product

$$
(f, g)_{\sim}=\int d k\left(1+\omega(k)^{-1}\right) f(k)^{*} g(k) .
$$

Then $\mathscr{A}$ is the CCR-algebra generated by the Weyl operators $W(f)$ with $f \in \tilde{\mathscr{H}}$. We regard $-\varepsilon \sigma_{x}$ as a perturbation of $H_{0}$, cf. (2.4). We define the state $\omega_{\beta}^{0}$ on $M_{2} \otimes \mathscr{A}$ by

$$
\omega_{\beta}^{0}=\left(\begin{array}{cc}
\left(1+e^{-2 \beta h}\right)^{-1} \omega_{+, \beta}^{0} & 0 \\
0 & \left(1+e^{2 \beta h}\right)^{-1} \omega_{-, \beta}^{0}
\end{array}\right),
$$

where $\omega_{ \pm, \beta}^{0}$ are the states on $\mathscr{A}$ given by

$\omega_{ \pm, \beta}^{0}(W(f))=\exp \left[-\frac{1}{2} \int d k|f(k)|^{2} \operatorname{coth}(\beta \omega(k) / 2) \pm \sqrt{\alpha / 2} \int d k \frac{\lambda(k)}{\omega(k)}\left(f(k)^{*}-f(k)\right)\right]$.

To $\omega_{\beta}^{0}$ there is associated the GNS-triplet $\left(\mathscr{H}_{\omega}, \pi_{\omega}, \Omega_{\omega}\right)$. For notational convenience, the algebra $M_{2} \otimes \mathscr{A}$ is identified with its representation. Let $\left(M_{2} \otimes \mathscr{A}\right)^{\prime \prime}$ be the von Neumann algebra generated by $M_{2} \otimes \mathscr{A}$. Since $H_{0}$ is the sum of two commuting quasifree Hamiltonians, the dynamics $\alpha_{t}^{0}$ generated by it on $\left(M_{2} \otimes \mathscr{A}\right)^{\prime \prime}$ is given explicitly. $\omega_{\beta}^{0}$ is a KMS state (equilibrium state) for $\alpha_{t}^{0}$. The full dynamics, $\alpha_{t}$, and the KMS state, $\omega_{\beta}$, of the spin-boson Hamiltonian $H$ is then constructed by a strongly convergent perturbation expansion in $\varepsilon[5,6]$. Note that in this fashion only a $W^{*}$-dynamical system is obtained $\left(\alpha_{t}\right.$ as defined is not a group of 
automorphisms on $M_{2} \otimes \mathscr{A}$ ). The ground states studied here are the limit of the states $\omega_{\beta}$ as $\beta \rightarrow \infty$.

$\omega_{\beta}$ is the thermal state without a Bose-Einstein condensate. $H_{0}$ could have other KMS states. They must have then a condensate with non-zero density. The perturbation of such states results in a thermal state of the spin-boson Hamiltonian with condensate. These other states are of no particular interest to us: Either (i) the condensate does not couple to the spin. This happens if $\lambda$ vanishes continuously at such $k$-values where $\omega(k)=0$. The thermal state is then a superposition of $\omega_{\beta}$ and the unperturbed condensate. Or (ii) the condensate couples to the spin. In the limit $\beta \rightarrow \infty$, this forces the spin to be either up or down with certainty. Thus, in the limit $\beta \rightarrow \infty$, we obtain a superposition of the unperturbed condensate and $\left(\begin{array}{cc}\omega_{+, \infty}^{0} & 0 \\ 0 & 0\end{array}\right)$ for $h>0$; respectively $\left(\begin{array}{cc}\frac{1}{2} \omega_{+, \infty}^{0} & 0 \\ 0 & \frac{1}{2} \omega_{-, \infty}^{0}\end{array}\right)$ for $h=0$. We briefly explain why at the end of Sect. 4.

Under some regularity assumptions, which in particular exclude condensation, Fannes, Nachtergaele and Verbeure [5] prove that $\omega_{\beta}$ is the unique KMS state of the spin-boson Hamiltonian.

The first result ensures the existence of ground states. Our real goal is an understanding of their qualitative properties. Existence is needed to set the arena.

Theorem 1. Let $\int d k \lambda(k)^{2}<\infty, \int d k \lambda(k)^{2} / \omega(k)<\infty$, and let $\omega_{\beta}$ be the equilibrium state at inverse temperature $\beta$ of the spin-boson Hamiltonian $H$ as constructed above. If needed the dependence on $\alpha, \varepsilon$ and $h$ is indicated as $\omega_{\beta, \alpha, \varepsilon, h}$. Convergence is understood in the weak ${ }^{*}$ sense on $\mathscr{A}$, i.e. $\omega_{\beta}(A)$ has a limit as $\beta \rightarrow \infty$ for all $A \in \mathscr{A}$.

(i) For $h \neq 0$ the limit

$$
\lim _{\beta \rightarrow \infty} \omega_{\beta, \alpha, \varepsilon, h}=\omega_{\infty, \alpha, \varepsilon, h}
$$

exists. The states in (2.10) have a limit as $h \rightarrow 0_{+}$and as $h \rightarrow 0_{-}$,

$$
\lim _{h \rightarrow \pm 0} \omega_{\infty, \alpha, \varepsilon, h}=\omega_{ \pm, \alpha, \varepsilon}\left(=\omega_{ \pm}\right) \text {. }
$$

$\omega_{+}$and $\omega_{-}$are related through the discrete symmetry $\tau, \tau \circ \tau=\mathbf{1}$, defined by

$$
\tau \sigma_{x}=\sigma_{x}, \quad \tau \sigma_{y}=-\sigma_{y}, \quad \tau \sigma_{z}=-\sigma_{z}, \quad \tau W(f)=W(-f) .
$$

(ii) We define the order parameter

$$
m^{*}=\omega_{+}\left(\sigma_{z}\right) .
$$

If $m^{*}=0$, then $\omega_{+}=\omega_{-}$. If $m^{*}>0$, then $\omega_{+} \neq \omega_{-}$.

(iii) If $h=0$ and $m^{*}=0$, then

$$
\lim _{\beta \rightarrow \infty} \omega_{\beta, \alpha, \varepsilon, 0}=\omega_{+, \alpha, \varepsilon}\left(=\omega_{-, \alpha, \varepsilon}\right) .
$$

If $h=0$ and $m^{*}>0$, then

$$
\lim _{\beta \rightarrow \infty} \omega_{\beta, \alpha, \varepsilon, 0}=\frac{1}{2} \omega_{+, \alpha, \varepsilon}+\frac{1}{2} \omega_{-, \alpha, \varepsilon}
$$

with the possible exception of a, at most, countable number of $\alpha$ 's (for fixed $\varepsilon$ ). 
Remark 1. The limit (2.15) should hold for all $\alpha$. Unfortunately, one can prove (2.15) only for such $\alpha$ 's, where the ground state energy is continuously differentiable in $\alpha$. By convexity there can be at most a countable number of exceptions.

As already indicated one could adopt other definitions of a ground state. A physically natural construction is the approximation through finite volume ground states. In position space we restrict the Bose field to the bounded region $\Lambda$ with suitable boundary conditions. The system has then a unique ground state. As $\Lambda \uparrow R^{d}$ sequence of ground states converges to a superposition of the states in Theorem 1 and, possibly, an unperturbed superfluid condensate.

There is also the algebraic definition of a ground state, cf. [6, Definition 5.3.18]. $\omega$ is an (algebraic) ground state, if

$$
\omega\left(A^{*}[H, A]\right) \geqq 0
$$

for all $A$ in the domain of the derivation $i[H, \cdot]$. Since $\alpha_{t}$ has been constructed only on $\left(M_{2} \otimes \mathscr{A}\right)^{\prime \prime}$ the definition, as it stands, is not applicable. A quite different problem is whether linear combinations of the states $\omega_{+}, \omega_{-}$exhaust already all algebraic ground states with no Bose-Einstein condensate. For the spin-boson Hamiltonian I conjecture that $\omega_{+}, \omega_{-}$are algebraic ground states and that there are no others. It would be of interest to elucidate this point.

To summarize: For $h=0$ and $m^{*}>0$, there are two distinct ground states, $\omega_{+}$ and $\omega_{-}$. The flip $(\tau-)$ symmetry of the spin-boson Hamiltonian is broken. With Theorem 1 we have established the general framework.

\section{Ground State Properties, Results}

We return to the ohmic case stated in the Introduction, i.e.

$$
\int d k \lambda(k)^{2}<\infty, \quad \lim _{t \rightarrow \infty} t^{2} W(t)=1 .
$$

To simplify notation we denote expectations with respect to $\omega_{ \pm, \alpha, \varepsilon}$ by $\langle\cdot\rangle_{ \pm}(\alpha, \varepsilon)$. $m^{*}(\alpha, \varepsilon)$ is increasing in $\alpha$ (and decreasing in $\varepsilon$ ). The critical coupling strength, $\alpha_{c}(\varepsilon)$, is then defined by

$$
\begin{array}{lll}
\left\langle\sigma_{z}\right\rangle_{+}(\alpha, \varepsilon)=0 & \text { for } & \alpha<\alpha_{c}(\varepsilon), \\
\left\langle\sigma_{z}\right\rangle_{+}(\alpha, \varepsilon)>0 & \text { for } \quad \alpha>\alpha_{c}(\varepsilon) .
\end{array}
$$

In fact $\left(3.2^{\prime}\right)$ holds also for $\alpha=\alpha_{c}(\varepsilon)$. We have the following qualitative bounds for the phase diagram.

\section{Theorem 2.}

(i) $\alpha_{c}(\varepsilon)$ is finite and increasing in $\varepsilon$.

(ii) The following lower bounds hold,

$$
\begin{aligned}
1 & \leqq \alpha_{c}(\varepsilon), \\
\left(\int_{0}^{\infty} d t W(t)\right)^{-1} \varepsilon & \leqq \alpha_{c}(\varepsilon) .
\end{aligned}
$$

(iii) One has the asymptotics 


$$
\begin{aligned}
\lim _{\varepsilon \rightarrow 0} \sup \alpha_{c}(\varepsilon) & \leqq 2, \\
\lim _{\varepsilon \rightarrow \infty} \frac{1}{\varepsilon} \alpha_{c}(\varepsilon) & =\left(\int_{0}^{\infty} d t W(t)\right)^{-1} .
\end{aligned}
$$

Remark 2. Equation (3.5) should read

$$
\lim _{\varepsilon \rightarrow 0} \alpha_{c}(\varepsilon)=1
$$

cf. Sect. 6 for details.

Remark 3. If

$$
\lim _{t \rightarrow \infty} t^{2} W(t)=0
$$

then $m^{*}=0$ for any coupling strength. Thus $\omega_{+}=\omega_{-}$. In fact the state $\langle\cdot\rangle_{+}$lies in Fock space. If

$$
\lim _{t \rightarrow \infty} t^{\gamma} W(t)=1, \quad 1<\gamma<2,
$$

then there is a critical coupling $\alpha_{c}$ such that $m^{*}>0$ for $\alpha>\alpha_{c}$. For small $\varepsilon$, $\alpha_{c}(\varepsilon) \cong \varepsilon^{(2-\gamma)}$, in particular $\alpha_{c}(\varepsilon) \rightarrow 0$ as $\varepsilon \rightarrow 0$ [2]. Thus the non-zero $\alpha_{c}\left(0_{+}\right)$is characteristic feature of the scale-invariant $1 / t^{2}$ interaction.

Theorem 3. The dichotomy

$$
\text { either }\left\langle\sigma_{z}\right\rangle_{+}=0 \quad \text { or } \quad \alpha\left\langle\sigma_{z}\right\rangle_{+}^{2} \geqq 1
$$

holds. In particular $\left\langle\sigma_{z}\right\rangle_{+}(\alpha, \varepsilon)$ jumps at least by $1 / \sqrt{\alpha_{c}(\varepsilon)}$ at $\alpha_{c}(\varepsilon)$.

Theorems 2 and 3 (and some further details given in [2]) in essence cover the ground state spin density matrix. Of interest are also properties of the Bose field. For $\alpha<\alpha_{c}$ the average spatial boson density decays integrably for large distances and the total number of bosons is finite. At $\alpha_{c}$ the average density jumps to a nonintegrable decay. The number of bosons is infinite with probability one. This is due to the generation of very many infrared bosons of small energy.

Theorem 4. For $h=0$ and $\alpha<\alpha_{c}$ the total number of bosons is finite,

$$
\int d x\left\langle a^{+}(x) a(x)\right\rangle_{+}(\alpha, \varepsilon)<\infty \text {. }
$$

On the other hand for $h=0$ and $\alpha \geqq \alpha_{c}$, the number of bosons is infinite.

Remark4. By (3.8) for $h=0$ and $\alpha<\alpha_{c}$, the ground state lies in Fock space. General positivity properties ensure that this is the only ground state in Fock space. Therefore $H$ as a self-adjoint operator in Fock space has a unique ground state for $h=0, \alpha<\alpha_{c}$ and has no ground state for $h=0, \alpha \geqq \alpha_{c}$.

\section{The Ising Representation of Thermal Expectations}

The Weyl operators satisfy the commutation relations

$$
W(f) W(g)=W(f+g) \exp \left[-\frac{1}{2} \int d k\left(f(k)^{*} g(k)-f(k) g(k)^{*}\right)\right],
$$




$$
W(f)^{*}=W(-f),
$$

$f \in \tilde{\mathscr{H}}$. In terms of the basic Bose operators

$$
W(f)=\exp \left[\int d k f(k) a^{+}(k)-\int d k f(k)^{*} a(k)\right] .
$$

The $W(f)$ 's are unitary, in particular $\|W(f)\|=1 . \mathscr{A}$ is the norm closure of $\{W(f) \mid f \in \tilde{\mathscr{H}}\}$. A state, $\omega$ on $M_{2} \otimes \mathscr{A}$ is determined by the expectations

$$
\omega(W(f)), \quad \omega\left(\sigma_{\zeta} W(f)\right), \quad \zeta=x, y, z
$$

for all $f$ in a dense subset of $\tilde{\mathscr{H}}$.

Thermal expectations of Weyl operators can be written as averages with respect to the one-dimensional Ising model of length $\beta$. The $\beta \rightarrow \infty$ limit corresponds to the infinite volume limit for the Ising model, which one (almost) knows how to control.

We have to introduce the Ising measures. For $\beta<\infty$ we need their finite volume version. A spin configuration, denoted by $\sigma$, on the interval $\Lambda$ is a function on $\Lambda$ which takes only the values $\pm 1 . n(\Delta)(\sigma)$ is the number of spin flips (= points of discontinuity) of the configuration $\sigma$ in the set $\Delta$. The space, $\Omega_{\Lambda}$, of all spin configurations over the interval $\Lambda$ is given by

$$
\Omega_{\Lambda}=\{t \mapsto \sigma(t) \in \pm 1, t \in \Lambda \mid n(\Delta)(\sigma)<\infty \text { for any bounded interval } \Delta \subset \Lambda\} .
$$

We choose $\sigma$ to be right continuous with left limits. Two configurations $\sigma, \sigma^{\prime}$ are close if $\sigma(t)=\sigma^{\prime}(t)$ except in the neighborhood of flip points. The Ising measures to be introduced below live on $\Omega_{[-\beta / 2, \beta / 2]}$.

Let

$$
W_{\beta}(t)=\int d k \lambda(k)^{2} \frac{1}{1-e^{-\beta \omega(k)}}\left(e^{-\omega(k) t}+e^{-\omega(k)(\beta-t)}\right),
$$

$0 \leqq t \leqq \beta . \mu_{[-\beta / 2, \beta / 2]}^{\varepsilon}(d \sigma)$ denotes the free spin measure over $[-\beta / 2, \beta / 2]$ with periodic boundary conditions. Its flip points are Poisson distributed in $[-\beta / 2, \beta / 2]$ with density $\varepsilon$ and are restricted to be even in number because of $\sigma(-\beta / 2)=\sigma(\beta / 2)$. The Ising measure of interest is given by

$$
\begin{gathered}
\frac{1}{Z} \mu_{[-\beta / 2, \beta / 2]}^{\varepsilon}(d \sigma) \exp \left[-\frac{\alpha}{8} \int_{-\beta / 2}^{\beta / 2} d t \int_{-\beta / 2}^{\beta / 2} d s W_{\beta}(|t-s|)(\sigma(t)-\sigma(s))^{2}\right. \\
\left.+h \int_{-\beta / 2}^{\beta / 2} d t \sigma(t)\right]=\langle\cdot\rangle_{\beta}(\alpha, \varepsilon, h) .
\end{gathered}
$$

If obvious from the context, $(\alpha, \varepsilon, h)$ will be omitted. We will also need the measure which has a flip at the origin with certainty. Let then $\langle\cdot\rangle_{\beta, 0}(\alpha, \varepsilon, h)$ denote the measure (4.6) conditioned that there is a spin flip at $t=0$.

Besides periodic boundary conditions also the standard,+- boundary conditions will be used. Note that in (4.6) the interaction is weakly $\beta$ dependent and tends to $W(t)$, cf. (1.3), as $\beta \rightarrow \infty$. The + state is defined with respect to the interaction $W(t)$, 


$$
\begin{aligned}
\langle\cdot\rangle_{+, T}= & \frac{1}{Z} \mu_{+,[-T, T]}^{\varepsilon}(d \sigma) \exp \left[-\frac{\alpha}{8} \int_{-T}^{T} d t \int_{-T}^{T} d s W(t-s)(\sigma(t)-\sigma(s))^{2}\right. \\
& \left.+h \int_{-T}^{T} d t \sigma(t)-\frac{\alpha}{8}\left(\int_{-\infty}^{-T} d t+\int_{T}^{\infty} d t\right)_{-T}^{T} d s W(t-s)(1-\sigma(s))^{2}\right] .
\end{aligned}
$$

Here $\mu_{[-T, T]}^{\varepsilon}(d \sigma)$ is the free spin-flip measure with $\sigma(-T)=1=\sigma(T),\langle\cdot\rangle_{+, T, x}$ denotes the Ising measure $\langle\cdot\rangle_{+, T}$ conditioned to have a flip at $x,|x|<T$.

The Weyl operators produce local external magnetic fields in the Ising model. For each $f \in \overline{\mathscr{H}}$ we define the external field

$$
\begin{aligned}
h_{f, \beta}(t)= & \frac{1}{2} \sqrt{\alpha / 2} \int d k \lambda(k)\left(f(k)+f(k)^{*}\right) \frac{1}{1-e^{-\beta \omega(k)}} \theta(t)\left(e^{-\omega(k)|t|}-e^{-\omega(k)(\beta-|t|)}\right) \\
& +\frac{1}{2} \sqrt{\alpha / 2} \int d k \lambda(k)\left(f(k)-f(k)^{*}\right) \frac{1}{1-e^{-\beta \omega(k)}}\left(e^{-\omega(k)|t|}+e^{-\omega(k)(\beta-|t|)}\right)
\end{aligned}
$$

with $|t| \leqq \beta / 2$. $\theta(t)=1$ for $t \geqq 0$ and $\theta(t)=-1$ for $t<0 . h_{f, \beta}(t)$ is bounded. The regular part of its derivative is

$$
\begin{aligned}
h_{f, \beta}^{\prime}(t)= & -\frac{1}{2} \sqrt{\alpha / 2} \int d k \lambda(k)\left(f(k)+f(k)^{*}\right) \frac{\omega(k)}{1-e^{-\beta \omega(k)}}\left(e^{-\omega(k)|t|}+e^{-\omega(k)(\beta-|t|)}\right) \\
& +\frac{1}{2} \sqrt{\alpha / 2} \int d k\left(f(k)-f(k)^{*}\right) \frac{\omega(k)}{1-e^{-\beta \omega(k)}} \theta(t)\left(e^{-\omega(k)|t|}-e^{-\omega(k)(\beta-|t|)}\right)
\end{aligned}
$$

with $|t| \leqq \beta / 2$.

Lemma 4.1. For arbitrary $\alpha, \varepsilon, h$ and every $f \in \tilde{\mathscr{H}}$ we have

$$
\begin{aligned}
& \omega_{\beta}(W(f))=\exp \left[-\frac{1}{2} \int d k|f(k)|^{2} \operatorname{coth}(\beta \omega(k) / 2]\right. \\
& \cdot\left\langle\exp \left[-\int_{-\beta / 2}^{\beta / 2} d t h_{f, \beta}(t) \sigma(t)\right]\right\rangle_{\beta} \\
& \omega_{\beta}\left(\sigma_{z} W(f)\right)=\exp \left[-\frac{1}{2} \int d k|f(k)|^{2} \operatorname{coth}(\beta \omega(k) / 2]\right. \\
& \cdot\left\langle\sigma(0) \exp \left[-\int_{-\beta / 2}^{\beta / 2} d t h_{f, \beta}(t) \sigma(t)\right]\right\rangle_{\beta}, \\
& \omega_{\beta}\left(\sigma_{y} W(f)\right)=-\frac{1}{2 i \varepsilon} \exp \left[-\frac{1}{2} \int d k|f(k)|^{2} \operatorname{coth}(\beta \omega(k) / 2)\right] \\
& \cdot\left(\int_{-\beta / 2}^{\beta / 2} d t h_{f, \beta}^{\prime}(t)\left\langle\sigma(0) \sigma(t) \exp \left[-\int_{-\beta / 2}^{\beta / 2} d t h_{f, \beta}(t) \sigma(t)\right]\right\rangle_{\beta}\right. \\
& \left.+\sqrt{\alpha / 2} \int d k \lambda(k)\left(f(k)+f(k)^{*}\right)\left\langle\exp \left[-\int_{-\beta / 2}^{\beta / 2} d t h_{f, \beta}(t) \sigma(t)\right]\right\rangle_{\beta}\right),
\end{aligned}
$$




$$
\begin{aligned}
\omega_{\beta}\left(\sigma_{x} W(f)\right)= & \exp \left[-\frac{1}{2} \int d k|f(k)|^{2} \operatorname{coth}(\beta \omega(k) / 2)\right] \\
& \cdot\left\langle\exp \left[-\int_{-\beta / 2}^{\beta / 2} d t h_{f, \beta}(t) \sigma(t)\right]\right\rangle_{\beta, 0}\left\langle\frac{1}{\delta} n([0, \delta])\right\rangle_{\beta}
\end{aligned}
$$

with $0<\delta<\beta / 2$.

Proof. We choose a finite mode approximation to $H$ avoiding the set $\{k \mid \omega(k)=0\}$, cf. e.g. [7, Sect. 5] for details. Then $H$ has a unique KMS state. Integrating out the bosons as in [2] results in the Ising expectations (4.10), (4.11) with $W_{\beta}(t)$ and $h_{\beta, f}(t)$ replaced by their finite mode approximation. As the number of modes tends to infinity, because of (2.1), the Ising expectations converge to (4.10), (4.11) and the finite mode KMS states converge to the state $\omega_{\beta}$ constructed through the perturbation expansion in $\varepsilon$.

An alternative route is to trotterize $\exp [-\beta H]$ with respect to the interaction $\sqrt{\alpha / 2} \sigma_{z} \int d k \lambda(k)\left(a^{+}(k)+a(k)\right)$. Let us use the short-hand $a^{\#}(f)=\int d k f(k) a^{\#}(k)$. Then averaging the product of Weyl operators yields

$$
\begin{aligned}
\exp & {\left[\frac{\alpha}{4} \int_{0}^{\beta} d t \int_{0}^{\beta} d s \sigma(t) \sigma(s) \mathscr{T}\left\langle\left[a^{+}(\lambda)+a(\lambda)\right]_{t}\left[a^{+}(\lambda)+a(\lambda)\right]_{s}\right\rangle\right.} \\
& +\sqrt{\alpha / 2} \int_{0}^{\beta} d t \sigma(t)\left\langle\left[a^{+}(\lambda)+a(\lambda)\right]_{t}\left[-a^{+}\left(f^{*}\right)+a(f)\right]\right\rangle \\
& \left.+\frac{1}{2}\left\langle\left[-a^{+}\left(f^{*}\right)+a(f)\right]^{2}\right\rangle\right] .
\end{aligned}
$$

Here $\mathscr{T}$ is the time-ordering. $\left\langle A_{t} A_{s}\right\rangle$ are imaginary time correlations with respect to $H^{0}=\int d k \omega(k) a^{+}(k) a(k)$, in particular,

$$
\langle W(f)\rangle=\exp \left[-\frac{1}{2} \int d k|f(k)|^{2} \operatorname{coth}(\beta \omega(k) / 2)\right] .
$$

In (4.10)-(4.13) we only shifted the time-interval to $[-\beta / 2, \beta / 2]$ in order to make the limit $\beta \rightarrow \infty$ more transparent.

Our construction covers (4.10), (4.11). To obtain (4.12) we use in addition that

$$
\frac{1}{2 i \varepsilon}\left[H, \sigma_{z}\right]=\sigma_{y}
$$

and therefore, by periodicity,

$$
\begin{aligned}
\omega_{\beta}\left(\sigma_{y} W(f)\right)= & \lim _{\delta \rightarrow 0} \frac{1}{\delta} \frac{1}{2 i \varepsilon}\left\langle\sigma ( 0 ) \left(\exp \left[-\int_{-\beta / 2}^{\beta / 2} d t h_{f, \beta}(t+\delta) \sigma(t)\right]\right.\right. \\
& \left.\left.-\exp \left[-\int_{-\beta / 2}^{\beta / 2} d t h_{f, \beta}(t) \sigma(t)\right]\right)\right\rangle_{\beta} \exp \left[-\frac{1}{2} \int d k|f(k)|^{2} \operatorname{coth}(\beta \omega(k) / 2)\right] .
\end{aligned}
$$

To obtain (4.13) we use $\sigma_{y} \sigma_{z}=i \sigma_{x}$ together with (4.16). Therefore

$$
\begin{gathered}
\omega_{\beta}\left(\sigma_{x} W(f)\right)=\lim _{\delta \rightarrow 0} \frac{1}{4 \delta} \frac{1}{\varepsilon}\left\langle(\sigma(\delta)-\sigma(0))^{2} \exp \left[-\int_{-\beta / 2}^{\beta / 2} d t h_{f, \beta}(t) \sigma(t)\right]\right\rangle_{\beta} \\
\cdot \exp \left[-\frac{1}{2} \int d k|f(k)|^{2} \operatorname{coth}(\beta \omega(k) / 2)\right]
\end{gathered}
$$


We want to prove that (4.11) to (4.13) have a limit as $\beta \rightarrow \infty$. In essence, this amounts to prove that a ferromagnetic Ising model with periodic boundary conditions has an infinite volume limit. The answer is well known. For $h \neq 0$ and for $h=0, m^{*}=0$, the limit is the unique Gibbs state. For $h=0$ and $m^{*}>0$, the limit is the $\frac{1}{2}$ mixture of the + and - state. This last statement can actually be proved only with the exception of a countable set of temperatures. In our context we have three complications: (i) The interaction and the magnetic fields are weakly $\beta$-(i.e. volume-) dependent. (ii) We have to show convergence also for the measure conditioned to have a flip at the origin. (iii) The space of configurations is not compact because of the arbitrary number of spin-flips in a bounded interval.

ad (i): This can be dealt with by simple uniform estimates. We do not take the space to write them out.

ad (ii): Fortunately the conditioning respects the FKG inequality. Thus the major part of the argument goes through without changes. Only at one point do we have to spatially average.

ad (iii) As is well known from the theory of stochastic processes, the convergence of moments has to be supplemented by tightness. We use reflection positivity to prove that the distribution of the number of spin flips in an arbitrary bounded interval decays faster than any exponential.

Let us introduce the infinite volume Ising measures. The limit $T \rightarrow \infty$ of the measures $\langle\cdot\rangle_{+(-), T}$, cf. (4.7), defines the probability measure $\langle\cdot\rangle_{+(-)}(\alpha, \varepsilon, h)$ $\left(=\langle\cdot\rangle_{+(-)}\right)$on $\Omega_{R}=\Omega$, the space of spin configurations over the real line. FKG domination ensures the existence of the limiting moments as $T \rightarrow \infty$. For $\langle\cdot\rangle_{+(-)}$ the number of spin flips in a bounded interval has a distribution which decays faster than any exponential. This follows from Lemma 4.4 to be proved below. As usual we define the spontaneous magnetization by

$$
m^{*}=\langle\sigma(0)\rangle_{+}(\alpha, \varepsilon, 0) .
$$

The conditioned measure $\langle\cdot\rangle_{+, T, x},|x|<T$, satisfies FKG. Spin flip at $x$ means $\sigma\left(x_{+}\right)=-\sigma\left(x_{-}\right)$. This can be achieved by a strong local magnetic field to the left of $x$ and an opposite one to the right of $x$. Thus FKG holds. By FKG domination the limit $T \rightarrow \infty$ of $\langle\cdot\rangle_{+, T, x}$ exists and defines the conditioned measure $\langle\cdot\rangle_{+, x}$ on $\Omega$. From Lemma 4.7 to be proved below it follows that for $\langle\cdot\rangle_{+, x}$ the distribution of the number of spin flips in a bounded interval decays faster than any exponential.

To state the limit $\beta \rightarrow \infty$ of (4.10) to (4.13) we have to define the local fields

$$
\begin{aligned}
h_{f}(t)= & \frac{1}{2} \sqrt{\alpha / 2} \int d k \lambda(k)\left(f(k)+f\left(k^{*}\right)\right) \theta(t) e^{-\omega(k)|t|} \\
& +\frac{1}{2} \sqrt{\alpha / 2} \int d k \lambda(k)\left(f(k)-f(k)^{*}\right) e^{-\omega(k)|t|}, \\
h_{f}^{\prime}= & -\frac{1}{2} \sqrt{\alpha / 2} \int d k \lambda(k)\left(f(k)+f(k)^{*}\right)^{\omega}(k) e^{-\omega(k)|t|} \\
& -\frac{1}{2} \sqrt{\alpha / 2} \int d k \lambda(k)\left(f(k)-f(k)^{*}\right) \omega(k) \theta(t) e^{-\omega(k)|t|} .
\end{aligned}
$$

Proposition 4.2.

(i) Let be either $h \neq 0$ or $h=0$ and $m^{*}=0$. Then for any $f \in \tilde{\mathscr{H}}$,

$$
\lim _{\beta \rightarrow \infty} \omega_{\beta}(W(f))=\exp \left[-\frac{1}{2} \int d k|f(k)|^{2}\right]\left\langle\exp \left[-\int d t h_{f}(t) \sigma(t)\right]\right\rangle_{+},
$$




$$
\begin{aligned}
\lim _{\beta \rightarrow \infty} \omega_{\beta}\left(\sigma_{z} W(f)\right)= & \exp \left[-\frac{1}{2} \int d k|f(k)|^{2}\right]\left\langle\sigma(0) \exp \left[-\int d t h_{f}(t) \sigma(t)\right]\right\rangle_{+}, \\
\lim _{\beta \rightarrow \infty} \omega_{\beta}\left(\sigma_{y} W(f)\right)= & -\frac{1}{2 i \varepsilon} \exp \left[-\frac{1}{2} \int d k|f(k)|^{2}\right] \\
& \cdot\left(\int d t h_{f}^{\prime}(t)\left\langle\sigma(0) \sigma(t) \exp \left[-\int d t h_{f}(t) \sigma(t)\right]\right\rangle_{+}\right. \\
& \left.+\sqrt{\alpha / 2} \int d k \lambda(k)\left(f(k)+f(k)^{*}\right)\left\langle\exp \left[-\int d t h_{f}(t) \sigma(t)\right]\right\rangle_{+}\right),
\end{aligned}
$$

$$
\begin{aligned}
\lim _{\beta \rightarrow \infty} \omega_{\beta}\left(\sigma_{x} W(f)\right)= & \exp \left[-\frac{1}{2} \int d k|f(k)|^{2}\right] \\
& \cdot\left\langle\exp \left[-\int d t h_{f}(t) \sigma(t)\right]\right\rangle_{+, 0}\left\langle\frac{1}{\delta} n([0, \delta])\right\rangle_{+} \cdot
\end{aligned}
$$

(ii) If $h=0$ and $m^{*}>0$, then for fixed $\varepsilon$ and with the possible exception of a countable set of $\alpha$ 's the limit $\beta \rightarrow \infty$ exists and is given by the expectations on the right-hand side of (4.22) to (4.25) with respect to the measure $\frac{1}{2}\langle\cdot\rangle_{+}+\frac{1}{2}\langle\cdot\rangle_{-}$.

To prove Proposition 4.2 we need some auxiliary lemmas.

Lemma 4.3. For $h \neq 0$ and for $h=0, m^{*}=0$, the moments converge,

$$
\lim _{\beta \rightarrow \infty}\left\langle\prod_{j=1}^{n} \sigma\left(t_{j}\right)\right\rangle_{\beta}=\left\langle\prod_{j=1}^{n} \sigma\left(t_{j}\right)\right\rangle_{+} .
$$

Proof. We use FKG domination and the Lee-Yang Theorem as in [8].

Lemma 4.4. Let $\Lambda \subset[-\beta / 2, \beta / 2]$ be a bounded interval and $z \in \mathbf{R}$. Then there is a constant $c(z)$, depending only on $z$, such that

$$
\left\langle e^{-z n(A)}\right\rangle_{\beta} \leqq e^{|\Lambda| c(z)} .
$$

Proof. Because of the underlying Hamiltonian the measure $\langle\cdot\rangle_{\beta}$ is reflection positive. This can also be verified directly from the definition of $\langle\cdot\rangle_{\beta}$. The free measure is reflection positive. The interaction can be written as

$$
\begin{gathered}
-\int_{0}^{\beta / 2} d t \int_{0}^{\beta / 2} d s W_{\beta}(|t-s|)(\sigma(t) \sigma(s)+\sigma(-t) \sigma(-s)) \\
-2 \int v(d \lambda) \int_{0}^{\beta / 2} d t e^{-\lambda t} \sigma(t) \int_{0}^{\beta / 2} d s e^{-\lambda s} \sigma(-s)
\end{gathered}
$$

with a positive measure $v$ because of (4.5). In this form [14] applies. Let $\Lambda=[0, t]$, $0<t<\beta / 2$. By reflection at zero and Schwarz inequality,

$$
\left\langle e^{-z n([0, t])}\right\rangle_{\beta} \leqq\left\langle e^{-z(n([0, t])+n([-t, 0]))}\right\rangle_{\beta}^{1 / 2} .
$$

We repeat (4.28) $n$ times such that $2^{n} t \leqq \beta$ but $2^{n+1} t>\beta$. If $z<0$, we bound $\exp \left[-z n\left(\left[-\beta / 2,-\beta / 2+2^{n} t\right]\right)\right] \leqq \exp [-z n([-\beta / 2, \beta / 2])]$. If $z>0$, we bound $\exp \left[-z n\left(\left[-\beta / 2,-\beta / 2+2^{n} t\right]\right)\right] \leqq \exp [-z n([-\beta / 2,0])]$ and apply (4.28) once more. In any case we obtain the bound 


$$
\left\langle e^{-z n([-\beta / 2, \beta / 2])}\right\rangle^{c|\Lambda| / \beta}=\exp \left[c|\Lambda| \frac{1}{\beta}\left(f_{\beta}(\varepsilon)-f_{\beta}\left(\varepsilon e^{-z}\right)\right)+c|\Lambda| \varepsilon\left(e^{-z}-1\right)\right]
$$

with the free energy $f_{\beta}(\varepsilon)=-\log Z, Z$ of (4.6).

As a prelude to the conditioned measure we need a result on the spin-flip density, $g_{+(-), T}(x)$, in the measure with $+(-)$ boundary conditions. $g_{+, T}(x)$ is defined by

$$
g_{+, T}(x)=\lim _{\delta \rightarrow \infty} \frac{1}{4 \delta}\left\langle[\sigma(x+\delta)-\sigma(x)]^{2}\right\rangle_{+, T}
$$

with $|x|<T$. Correspondingly we define $g_{-, T}(x) . g$ is the infinite volume spin-flip density. By translation invariance it is constant. Thus $g$ is defined either as in (4.30) or by

$$
g=\frac{1}{\delta}\langle n([0, \delta])\rangle_{+},
$$

independently of $\delta, \delta>0 . g$ agrees for + and - boundary conditions.

We note that

$$
\int_{-\delta}^{\delta} d x g_{+, T}(x)=\langle n([-\delta, \delta])\rangle_{+, T}
$$

Hence

$$
\lim _{T \rightarrow \infty} \int_{-\delta}^{\delta} d x g_{+, T}(x)=2 \delta g .
$$

We have to strengthen the convergence.

Lemma 3.5. We have

$$
\lim _{T \rightarrow \infty} g_{+, T}(x)=g
$$

uniformly on compact intervals.

Proof. For $|x|<T$ we differentiate and obtain

with

$$
\frac{d}{d x} g_{+, T}(x)=\alpha_{T}(x) g_{+, T}(x)
$$

$$
\begin{gathered}
\alpha_{T}(x)=2\left\langle\left(h+h_{T}(x)+\frac{\alpha}{2} \int_{-T}^{T} d t W(t-x) \sigma(t)\right) \theta_{x}(\sigma)\right\rangle_{+, T, x} \\
h_{T}(x)=\frac{\alpha}{2}\left(\int_{-\infty}^{-T} d t W(t-x)+\int_{T}^{\infty} d t W(t-x)\right) .
\end{gathered}
$$

Here $\langle\cdot\rangle_{+, T, x}$ is the measure $\langle\cdot\rangle_{+, T}$ conditioned to have a flip at $x . \theta_{x}=1$ if $\sigma\left(x_{-}\right)=1$ and $\theta_{x}=-1$ if $\sigma\left(x_{-}\right)=-1$. Clearly

$$
\left|\alpha_{T}(x)\right| \leqq c \text {. }
$$


Now let $[-\delta, \delta]$ be the interval of interest. Then

$$
\int_{-\delta}^{\delta} d x g_{+, T}(x)=\int_{-\delta}^{\delta} d x \exp \left[\int_{y}^{x} d x^{\prime} \alpha_{T}\left(x^{\prime}\right)\right] g_{+, T}(y),
$$

$|y| \leqq \delta$. By (4.39) and (4.36) we conclude that

$$
\sup _{|x| \leqq \delta} g_{+, T}(x) \leqq c, \quad \sup _{|x| \leqq \delta}\left|\frac{d}{d x} g_{+, T}(x)\right| \leqq c,
$$

uniformly in $T$. Together with (4.33) this implies the assertion

Lemma 3.6. For $h \neq 0$ and for $h=0, m^{*}=0$, the moments converge

$$
\lim _{\beta \rightarrow \infty}\left\langle\prod_{j=1}^{n} \sigma\left(t_{j}\right)\right\rangle_{\beta, 0}=\left\langle\prod_{j=1}^{n} \sigma\left(t_{j}\right)\right\rangle_{+, 0} .
$$

Proof. By FKG domination (4.41) holds provided that

$$
\langle\sigma(t)\rangle_{+, 0}=\langle\sigma(t)\rangle_{-, 0}
$$

for all $t \neq 0$. We choose a sufficiently small $\delta$ and average,

$$
\begin{aligned}
\int_{-\delta}^{\delta} & d x\langle\sigma(t-x)\rangle_{+, T, 0} \\
& =\int_{-\delta}^{\delta} d x\langle\sigma(t)\rangle_{+,[-T+x, T+x], x} \\
& \leqq \int_{-\delta}^{\delta} d x\langle\sigma(t)\rangle_{+,[-T+\delta, T-\delta], x} \\
& =\int_{-\delta}^{\delta} d x \lim _{\lambda \rightarrow 0} \frac{1}{\lambda}\left\langle\sigma(t)[\sigma(x+\lambda)-\sigma(x)]^{2}\right\rangle_{+, T-\delta} / g_{+, T-\delta}(x) .
\end{aligned}
$$

By Lemma $4.5 g_{+, T-\delta}(x)$ converges uniformly to $g$. Therefore (4.43) is bounded by

$$
\frac{1}{g-(1 / T)}\langle\sigma(t) n([-\delta, \delta])\rangle_{+, T-\delta}
$$

for sufficiently large $T$. Repeating the same argument for the-boundary condition we obtain, for $T$ sufficiently large,

$$
\begin{aligned}
& \frac{1}{g+(1 / T)}\langle\sigma(t) n([-\delta, \delta])\rangle_{-, T+\delta} \\
& \leqq \int_{-\delta}^{\delta} d t\langle\sigma(t)\rangle_{-, T, 0} \leqq \int_{-\delta}^{\delta} d t\langle\sigma(t)\rangle_{+, T, 0} \\
& \leqq \frac{1}{g-(1 / T)}\langle\sigma(t) n([-\delta, \delta])\rangle_{+, T-\delta} .
\end{aligned}
$$

By Lemma 4.3 and 4.4 the upper and lower bounds agree as $T \rightarrow \infty$. Thus (4.42) holds $t$ - almost surely. By spectral representation $\langle\sigma(t)\rangle_{0,+}$ is continuous in $t$ 
Lemma 4.7. Let $\Lambda \subset[-\beta / 2, \beta / 2]$ be a bounded interval. Then for any $z<0$ there is a constant $c_{0}(z)$ such that

$$
\left\langle e^{-z n(\Lambda)}\right\rangle_{\beta, 0} \leqq c_{0}(z)
$$

uniformly in $\beta$.

Proof. Let $g_{\beta}$ be the spin-flip density for $\langle\cdot\rangle_{\beta}$. Let $\Lambda^{\delta}$ be the interval $\Lambda$ with a border of length $\delta$ added. Then, using periodicity,

$$
\begin{aligned}
\left\langle e^{-z n(\Lambda)}\right\rangle_{\beta, 0}= & \frac{1}{2 \delta} \int_{-\delta}^{\delta} d x\left\langle e^{-z n(\Lambda+x)}\right\rangle_{\beta, x} \leqq \frac{1}{2 \delta} \int_{-\delta}^{\delta} d x\left\langle e^{-z n\left(\Lambda^{\delta}\right)}\right\rangle_{\beta, x} \\
& =\left\langle e^{-z n\left(\Lambda^{\delta}\right)} \frac{1}{2 \delta} n([-\delta, \delta])\right\rangle_{\beta} / g_{\beta} .
\end{aligned}
$$

The claim follows now by Schwarz inequality and Lemma 4.4

Proof of Proposition 4.2. The differences

and

$$
\int_{-\beta / 2}^{\beta / 2} d t \int_{-\beta / 2}^{\beta / 2} d s\left(W_{\beta}(t-s)-W(t-s)\right)(\sigma(t)-\sigma(s))^{2}
$$

$$
\int_{-\beta / 2}^{\beta / 2} d t\left(h_{f, \beta}(t)-h_{f}(t)\right) \sigma(t)
$$

tend to zero as $\beta \rightarrow \infty$ uniformly in $\sigma$.

ad (i): Lemma 4.3 and 4.4 for $\langle\cdot\rangle_{\beta}$ respectively Lemma 4.6 and 4.7 for $\langle\cdot\rangle_{\beta, 0}$, ensure the convergence for averages over local functions as in (4.10) to (4.12), respectively as in (4.13).

ad (ii): If $h=0$ and $m^{*}>0$ then $\langle\cdot\rangle_{+} \neq\langle\cdot\rangle_{-}$. Tightness holds as before. The problem is to identify the limit(s) of the sequences $\langle\cdot\rangle_{\beta}$ and $\langle\cdot\rangle_{\beta, 0}$.

Let us assume that

$$
\int d t W(t)\langle\sigma(0) \sigma(t)\rangle_{+}(\alpha, \varepsilon, 0)
$$

is continuous at $\alpha$. By convexity of the ground state energy ( $\hat{=}$ Ising free energy) in $\alpha$, this holds for all $\alpha \geqq \alpha_{c}$ except possibly a countable set. By tightness, we can choose a subsequence such that $\langle\cdot\rangle_{\beta}$ has a limit measure $\langle\cdot\rangle$, on $\Omega$ as $\beta \rightarrow \infty$. $\langle\cdot\rangle$ is translation invariant [9], cf. also [10], and hence $\int d t W(t)\langle\sigma(t) \sigma(0)\rangle_{+}=$ $\int d t W(t)\langle\sigma(t) \sigma(0)\rangle$. Since $W(t),\langle\sigma(t) \sigma(0)\rangle_{+},\langle\sigma(0) \sigma(t)\rangle$ are positive, we have $\langle\sigma(t) \sigma(0)\rangle_{+}=\langle\sigma(t) \sigma(0)\rangle$. By [9] all even correlation functions must agree. Since for $\langle\cdot\rangle_{\beta}$, hence also for $\langle\cdot\rangle$, all odd correlation functions vanish, we must have $\langle\cdot\rangle=\frac{1}{2}\langle\cdot\rangle_{+}+\frac{1}{2}\langle\cdot\rangle_{-}$.

For the conditioned measure we average, as before, over a small interval. Then

$$
\int_{-\delta}^{\delta} d x\left\langle\prod \sigma\left(t_{j}-x\right)\right\rangle_{\beta, 0}=\left\langle\prod \sigma\left(t_{j}\right) n([-\delta, \delta])\right\rangle_{\beta} / g_{\beta} .
$$

The right-hand side converges to

$$
\int_{-\delta}^{\delta} d x\left(\frac{1}{2}\left\langle\prod \sigma\left(t_{j}-x\right)\right\rangle_{+, 0}+\frac{1}{2}\left\langle\prod \sigma\left(t_{j}-x\right)\right\rangle_{-, 0}\right),
$$


which identifies the limit measure for $\langle\cdot\rangle_{\beta, 0}$ as $\beta \rightarrow \infty$

By definition the state $\omega_{\beta}$ has no superfluid phase. Let us try to understand then how the spin-boson system behaves in the presence of a condensate. For the sake of discussion we assume that $\omega$ and $\lambda$ are continuous, $\omega(0)=0$ and $\omega(k)$ strictly bounded away from zero otherwise. The average superfluid density, $\rho_{s}$, depends on how the limit of infinite volume, i.e. of infinitely many modes, is carried out. However, if this limit exists at all, because of (4.14), the functional integral for $\omega_{\beta}(W(f))$ must acquire the extra piece

$$
\exp \left[\frac{1}{2} \rho_{s}\left(\frac{\alpha}{2} \lambda(0)^{2}\left(\int_{0}^{\beta} d t \sigma(t)\right)^{2}+2 \lambda(0) \sqrt{\alpha / 2}\left(f(0)-f(0)^{*}\right) \int_{0}^{\beta} d t \sigma(t)-|f(0)|^{2}\right)\right],
$$

$f$ continuous close to $k=0$. If $\lambda(0)=0$, then the condensate does not couple to the spin. If $\lambda(0) \neq 0, \rho_{s}>0$, then the long range term

$$
\int_{0}^{\beta} d t \int_{0}^{\beta} d s \sigma(t) \sigma(s)
$$

dominates for $\beta \rightarrow \infty$, since it is proportional to $\beta^{2}$ rather than $\beta$. The long range term forces the spin to be either up or down with probability one.

We do not attempt to give a full proof here. Rather let us explain the case of a pure condensate, denoted by $\omega_{\beta}^{*}$. Then, undoing the square,

$$
\begin{aligned}
\omega_{\beta}^{*}(W(f))= & \exp \left[-\frac{1}{2} \rho_{s}|f(0)|^{2}\right]\left\{\int d \xi e^{-\xi^{2} / 2 D} \int \mu_{[0, \beta]}^{\varepsilon}(d \sigma)\right. \\
& \cdot \exp \left[(\xi+h+i \gamma) \int_{0}^{\beta} d t \sigma(t)\right] / \int d \xi e^{-\xi^{2} / 2 D} \int \mu_{[0, \beta]}^{\varepsilon}(d \sigma) \\
& \left.\cdot \exp \left[(\xi+h) \int_{0}^{\beta} d t \sigma(t)\right]\right\},
\end{aligned}
$$

where $D=\rho_{s} \alpha \lambda(0)^{2} / 2$ and $\gamma=\lambda(0) \sqrt{\alpha / 2} \operatorname{Im} f(0)$. The integration over $\mu(d \sigma)$ yields $\cosh \left(\beta \sqrt{\left.\varepsilon^{2}+(\xi+h+i \gamma)^{2}\right)}\right.$. For large $\beta$ the $\xi$ integral is estimated by steepest descent. Since the functions are given explicitly, it is not hard to show that the error term vanishes as $\beta \rightarrow \infty$. As a net result the term in the curly brackets tends to one as $\beta \rightarrow \infty$. The corresponding computation for $\omega_{\beta}^{*}\left(\sigma_{\zeta} W(f)\right), \zeta=x, y, z$, yields

$$
\lim _{\beta \rightarrow \infty} \exp \left[\frac{1}{2} \rho_{s}|f(0)|^{2}\right] \omega_{\beta}^{*}\left(\sigma_{\zeta} W(f)\right)=\left\{\begin{array}{ll}
0 & \text { for } \zeta=x, y \quad \text { and } \zeta=z, h=0 \\
\operatorname{sign} h & \text { for } \zeta=z, h \neq 0
\end{array} .\right.
$$

Thus, in the limit $\beta \rightarrow \infty$, the spin decouples from the condensate. If $h>0(<0)$, the spin is up (down). For $h=0$ the spin state is a $\frac{1}{2}$ mixture of up and down.

\section{The Fortuin-Kasteleyn Representation and Continuum Percolation}

Symmetry breaking for the spin-boson Hamiltonian is equivalent to the existence of a ferromagnetic phase transition for the one-dimensional continuum Ising model 
with long range, $1 / t^{2}$, interactions. Thus we have to analyze the phase-diagram of the Ising model in the $(\alpha, \varepsilon)$-plane. Since the results of [4] refer to the standard Ising model over $Z$, an immediate impulse is to use a discrete approximation to the continuum model. We choose a lattice spacing $\delta$. The Ising model approximating (4.7) as $\delta \rightarrow 0$ has then

(i) a nearest neighbor coupling of strength $\frac{1}{2}|\log \varepsilon \delta|$

(ii) a long range coupling of strength $(\alpha / 2) \delta^{2} W((i-j) \delta), i, j \in Z$.

We note that the asymptotics of the long range part,

$$
\lim _{j \rightarrow \infty} j^{2}\left(\delta^{2} W(j \delta)\right)=1
$$

independent of the lattice spacing. On the other hand the nearest neighbor coupling is increasing whereas the long range couplings are decreasing as $\delta \rightarrow 0$. For this reason we do not know whether there is monotinicity with respect to $\delta$.

Let $\langle\cdot\rangle_{+}^{\delta}$ be the infinite volume Ising measure for the potentials (i), (ii) above with + boundary conditions on a lattice with lattice spacing $\delta$. In the obvious way, we may think of $\langle\cdot\rangle_{+}^{\delta}$ as a measure on $\Omega$. Tightness can be shown as before, using reflection positivity. Therefore there exists a subsequence such that, weakly on $\Omega,\langle\cdot\rangle_{+}^{\delta}$ has a limit as $\delta \rightarrow 0$. Let us denote the limit measure by $\langle\cdot\rangle$. Since $\langle\cdot\rangle^{\delta}{ }_{+}$is translation invariant, so is $\langle\cdot\rangle$. The limit measure $\langle\cdot\rangle$ has to be a Gibbs measure for the potentials $h$ and $(\alpha / 2) W(t)$, because the conditional measures for $\langle\cdot\rangle$ satisfy the DLR equations with respect to these potenials. If $h \neq 0$, then the continuum model has a unique Gibbs measure. Hence

$$
\lim _{\delta \rightarrow \infty}\langle\cdot\rangle_{+}^{\delta}(\alpha, \varepsilon, h)=\langle\cdot\rangle_{+}(\alpha, \varepsilon, h),
$$

$h \neq 0$. We expect (5.1) also to hold for $h=0$. Unfortunately we do not know how to show this from abstract arguments.

In order to prove Theorems 2 to 4 we are forced then to pay closer attention to the proofs in $[3,4]$. Their essential tool is the Fortuin-Kasteleyn (FK) representation which leads to a percolation problem with long range bonds. This construction works also here giving rise to a continuum percolation problem. Incidentally Aizenman and Newman [3] also studied a continuum percolation bearing some similarity to ours.

We work in finite volume $[-T, T]$ with + boundary conditions. The external field $h=0$. For the discrete approximation, lattice spacing $\delta$, the FK representation yields the following bond percolation:

(i) Nearest neighbor bonds $\{j \delta,(j+1) \delta\}$ are occupied with probability $1-\varepsilon \delta$, empty with probability $\varepsilon \delta$.

(ii) General bonds $\{l \delta, j \delta\}, i<j$, are occupied with probability $1-e^{-\delta^{2} \alpha W((i-j) \delta)}$, empty with probability $e^{-\delta^{2} \alpha W((i-j) \delta)}$.

The bond variables are independent. To obtain the Ising model each bond configuration is weighted by

$$
\text { (independent weight) } \times q^{C_{T}},
$$


$q=2$, where $C_{T}$ is number of connected components. Here $(-\infty,-T] \cup[T, \infty)$ is considered as a single connected component. This is the proper transcription of the + boundary conditions.

As $\delta \rightarrow \infty$ the discrete Ising model tends to its continuum version (4.7). Clearly also the bond percolation has a well defined limit:

(i) On the real line we have a locally finite configuration of points. We refer to them as points of fracture. A configuration of points of fracture is denoted by $\tau=\left\{\tau_{j} \mid j=0, \pm 1, \ldots\right\}, \tau_{j} \leqq \tau_{j+1}$. For independent percolation the points of fracture have a Poisson distribution with constant density $\varepsilon$.

(ii) On the half-plane $\mathbf{R}_{0}^{2}=\{s, t \mid s \leqq t\}$ we have a locally finite configuration of points denoted by $\omega=\left\{\omega_{j} \mid j=1,2, \ldots\right\}, \omega_{j}=\left\{s_{j}, t_{j}\right\}$. Each point $\left\{s_{j}, t_{j}\right\}$ is referred to as bond starting from the site $s_{j}$ and reaching the site $t_{j}, s_{j} \leqq t_{j}$. For independent percolation the bonds are distributed according to an inhomogeneous Poisson process with density $\alpha W(t-s) d t d s$. Bonds and points of fracture are independent.

The open intervals $\left(\tau_{j}, \tau_{j+1}\right), j=0, \pm 1, \ldots$, form the basic components. Two intervals, $\left(\tau_{i}, \tau_{i+1}\right)$ and $\left(\tau_{j}, \tau_{j+1}\right)$ belong to the same connected component if there exists a bond $\{s, t\}$ such that $s \in\left(\tau_{i}, \tau_{i+1}\right)$ and $t \in\left(\tau_{j}, \tau_{j+1}\right)$. The bonds of (ii) link the basic components. This concludes our prescription for the independent continuum percolation.

To obtain the correlated percolation corresponding to the Ising model we have to go through the finite volume construction. Let $\tau_{-}$be the smallest point of fracture with $-T \leqq \tau_{-}$and $\tau_{+}$be the largest point of fracture with $\tau_{+} \leqq T$. Let $C_{T}(\tau, \omega)$ be the number of connected components in the configuration $(\tau, \omega)$, where $\left(-\infty, \tau_{-}\right) \cup\left(\tau_{+}, \infty\right)$ is taken as a single component. In the finite volume $[-T, T]$ the configuration $(\tau, \omega)$ is weighted by

$$
\text { (independent weight) } \times q^{C_{T(\tau, \omega)}},
$$

$q \geqq 1$. Independent percolation is $q=1$ and Ising means $q=2$. Normalized to one (5.4) defines a point process on $\mathbf{R} \cup \mathbf{R}_{0}^{2}$. Let $\mathbf{P}_{q, T}$ denote the corresponding probability measure. Again the object of interest is its infinite volume limit

$$
\lim _{T \rightarrow \infty} \mathbf{P}_{q, T}=\mathbf{P}_{q} \text {. }
$$

The limit exists by monotinicity in the FKG sense.

The FKG property is an important tool for analyzing the correlated percolation. Therefore let us summarize the main points. Let $\Gamma$ denote the set of all locally finite point configurations on $\mathbf{R} \cup \mathbf{R}_{0}^{2}$. We introduce a partial order between configurations by

$$
(\tau, \omega) \prec\left(\tau^{\prime}, \omega^{\prime}\right),
$$

$\left(\tau^{\prime}, \omega^{\prime}\right)$ dominates $(\tau, \omega)$, if as point sets $\tau \supset \tau^{\prime}$ and $\omega \subset \omega^{\prime}$. A function on $\Gamma$ is said to be increasing if it is non-decreasing with respect to this partial order. A probability measure, $\mathbf{P}$ on $\Gamma$ is an FKG measure if

$$
\mathbf{P}(f g) \geqq \mathbf{P}(f) \mathbf{P}(g)
$$


for all increasing, bounded and measurable functions $f, g$ on $\Gamma$. For two measures $\mathbf{P}, \mathbf{P}^{\prime}$ on $\Gamma, \mathbf{P}^{\prime}$ dominates $\mathbf{P}$ in the FKG sense, $\mathbf{P}^{\prime}>\mathbf{P}$, if

$$
\mathbf{P}^{\prime}(f) \geqq \mathbf{P}(f)
$$

for all increasing, bounded, and measurable functions $f$ on $\Gamma$.

In [4] it is shown that the lattice version of the random cluster model is FKG and that the measures for + boundary conditions decrease as $T \rightarrow \infty$. These properties carry over to the continuum percolation. Thus $\mathbf{P}_{q, T}$ and $\mathbf{P}_{q}$ are FKG measures, $q \geqq 1$, and the domination

$$
\mathbf{P}_{q, T}>\mathbf{P}_{q, T^{\prime}}
$$

with $T^{\prime} \geqq T$ holds.

There are two other, most useful comparison inequalities with respect to $q, W$ and $\varepsilon$. If $q^{\prime} \geqq q \geqq 1$ and $\alpha^{\prime} W^{\prime}(t) \leqq \alpha W(t), \varepsilon^{\prime} \geqq \varepsilon$, then

$$
\mathbf{P}_{q} \succ \mathbf{P}_{q^{\prime}}^{\prime} .
$$

If $q^{\prime} \geqq q \geqq 1$ and $\alpha^{\prime} W^{\prime}(t) / q^{\prime} \geqq \alpha W(t) / q, \varepsilon^{\prime} q^{\prime} \leqq \varepsilon q$, then

$$
\mathbf{P}_{q} \prec \mathbf{P}_{q^{\prime}}^{\prime} .
$$

If we choose $q=1$, then the correlated percolation is compared with independent percolation. From this we can read off regularity properties of $\mathbf{P}_{q}$. In particular, the number of points in any bounded region of $\mathbf{R} \cup \mathbf{R}_{0}^{2}$ has a distribution which decays faster than any exponential. Also, all accidental degeneracies have measure zero. Thus, by discarding a set of measure zero, we may assume that $\tau_{j}<\tau_{j+1}$, $s_{j}<t_{j}$ and $s_{i} \neq \tau_{i}, \tau_{j} \neq \tau_{j}$ for all $i, j$.

Let $A$ be an event defined by specifying the configuration in an arbitrary subset of $\mathbf{R} \cup \mathbf{R}_{0}^{2}$. Then also the conditional measures $\mathbf{P}_{q, T}(\cdot \mid A)$ and $\mathbf{P}_{q}(\cdot \mid A)$ enjoy the FKG property. It remains to state the correspondence with the quantities of interest. We have

$$
\begin{aligned}
m^{*} & =\langle\sigma(0)\rangle_{+}=\mathbf{P}_{2}(\{0 \text { belongs to an infinite cluster }\}), \\
\langle\sigma(0) \sigma(t)\rangle_{+} & =\mathbf{P}_{2}(\{0, t \text { belong to the same connected component }\}) .
\end{aligned}
$$

\section{Bounds on the Critical Coupling}

We prove Theorem 2

Proof of Theorem 2 (i). The existence of $\alpha_{c}(\varepsilon)$ is proved in [2]. Decreasing $\varepsilon$ increases the nearest neighbor coupling. By Griffiths inequality $\langle\sigma(0)\rangle_{+}(\alpha, \varepsilon)$ is decreasing in $\varepsilon$ and hence $\alpha_{c}(\varepsilon)$ is increasing

Proof of Theorem 2 (ii). The lower bound (3.4) is the mean field bound [2].

This bound misses the important qualitative feature that $\alpha_{c}(\varepsilon)$ tends to a limit larger than zero as $\varepsilon \rightarrow 0$. To prove that $1 \leqq \alpha_{c}(\varepsilon)$ we need the machinery of [3]. In fact, $1 \leqq \alpha_{c}(\varepsilon)$ follows from the dichotomy (3.7) since $\left\langle\sigma_{z}\right\rangle_{+} \leqq 1$. Nevertheless it is useful to study the simpler problem (3.3) first. It exemplifies the changes needed in the proof of (3.7) as compared to [3]. Actually, they are surprisingly minor. 
Let $\alpha_{c, 1}(\varepsilon)$ be the critical coupling strength for the independent percolation $\mathbf{P}_{1}$ and $m_{1}^{*}$ be its percolation probability. Since by $(5.10) m^{*} \leqq m_{1}^{*}$, we have

$$
\alpha_{c, 1}(\varepsilon) \leqq \alpha_{c}(\varepsilon) \text {. }
$$

Hence it suffices to show that, if $\alpha<1$, the independent model does not percolate.

Because of the points of fracture we have to redefine the points of "furthest direct reach," cf. [3, Sect. 2]. We consider the stochastic process $\left\{L_{n}, n \geqq 0\right\}$ with $L_{0}=0, L_{1}$ the smallest point of fracture, $\tau_{j}$, such that $\tau_{j-1}<L<\tau_{j}$, and for $n>1$,

$$
L_{n+1}= \begin{cases}\text { largest point of fracture, } \tau_{j}, \text { such there } & \\ \text { is a bond between some } x \in\left(L_{n-1}, L_{n}\right) & \\ \text { and } y \in\left(\tau_{j-1}, \tau_{j}\right), L_{n} \leqq \tau_{j-1}, & \text { if there is such a point } \\ L_{n}, & \text { otherwise. }\end{cases}
$$

The increments are $U_{n}=L_{n}-L_{n-1}$.

Because of independence the conditional probabilities for the increments can be obtained explicitly,

$$
\begin{aligned}
\mathbf{P}_{1}\left(U_{n+1} \leqq\right. & \left.u \mid U_{1}, \ldots, U_{n}\right)=e^{-\varepsilon u} \exp \left[-\alpha \int_{-U_{n}}^{0} d s \int_{0}^{\infty} d t W(t-s)\right] \\
& +\int_{0}^{u} d \xi \varepsilon e^{-\varepsilon \xi} \exp \left[-\alpha \int_{-U_{n}}^{0} d s \int_{u-\xi}^{\infty} W(t-s)\right] \geqq\left(1+U_{n} / u\right)^{-\alpha},
\end{aligned}
$$

if $u \geqq D^{+}$with $D^{+}$sufficiently large.

In the definition of the set $A_{L, K}$ we add the requirement that $\xi_{+}$and $\xi_{-}$are points of fracture. In the definition of the set $F_{L, K}$ we require that $\xi$ is a point of fracture. It is advantageous not to impose such a requirement for the left endpoint 0 .

With these changes Lemma 3.1 of [3] holds. The remainder of the argument carries over unaltered: only events referring to bonds are estimated. The information on the points of fracture are contained in ${ }^{\wedge} F_{V, r}$ and ${ }^{\wedge} F_{V, r}^{*}$, which are estimated by [3, Lemma 3.1]

Proof of Theorem 2 (iii). (small $\varepsilon$ ) Let $m_{1}^{*}(\alpha, \varepsilon)$ be the percolation probability for the independent percolation $\mathbf{P}_{1}$. By (5.10) we have

$$
m_{1}^{*}(\alpha / 2,2 \varepsilon) \leqq m^{*}(\alpha, \varepsilon)
$$

We have to show that for any $\alpha>2$ we can achieve $m_{1}^{*}>0$ by making $\varepsilon$ small enough. We scale distances by a factor $2 \varepsilon$. Then the density of points on the line is 1 and the bond density is $(\alpha / 2)(2 \varepsilon)^{-2} W((t-s) / 2 \varepsilon)$. To $\left\{\tau_{j}\right\}$ we superimpose the eually spaced configuration $\{n, n \in \mathbf{Z}\}$ of points of fracture. Using only bonds with $s, t \in(n, n+1)$, an open interval $(n, n+1)$ is a single connected component with probability $p_{\varepsilon}$. The intervals $(m, m+1)$ and $(n, n+1)$ are connected at least with probability

$$
p(n-m)=p_{\varepsilon} p_{\varepsilon}\left(1-\exp \left[-\frac{\alpha^{m+1}}{2} \int_{m}^{n} d s \int_{n}^{n+1} d t(2 \varepsilon)^{-2} W((t-s) / 2 \varepsilon)\right]\right)
$$


$m<n$. Thus (6.5) defines a long range independent percolation problem on $\mathbf{Z}$. Its percolation probability is a lower bound for $m_{1}^{*}(\alpha / 2,2 \varepsilon)$.

Using that the points in $[n, n+1]$ and the bonds are independent and that both have a Poisson statics, we bound $p_{\varepsilon}$ by

$$
1-p_{\varepsilon} \leqq \int_{0}^{1} d q \exp \left[-\frac{\alpha}{2} \int_{0}^{q} d s \int_{q}^{\infty} d t(2 \varepsilon)^{-1} W((t-s) / 2 \varepsilon)\right]
$$

Therefore $p_{\varepsilon} \rightarrow 1$ as $\varepsilon \rightarrow 0$. For large $n-m, p(n-m) \simeq p_{\varepsilon}^{2}(\alpha / 2)|n-m|^{-2}$. The nearest neighbor probability, $p(1)$, tends to 1 as $\varepsilon \rightarrow 0$. If $\alpha / 2>1$, [11, Theorem 1.2] asserts that the system percolates provided $\varepsilon$ is small enough

Remark. Imbrie and Newman [12] proved recently that the standard Ising model with pair interaction $(\alpha / 2)|i-j|^{-2}$ for large separation has a non-zero spontaneous magnetization provided $\alpha>1$ and the nearest neighbor coupling is sufficiently strong. Clearly, for the spin-boson Hamiltonian we expect then $\lim _{\varepsilon \rightarrow 0} \alpha_{c}(\varepsilon)=1$.

We lost a factor 2 by estimating $m^{*}$ by $m_{1}^{*}$. Let us try to use the same tecnique as for large $\varepsilon$. The flip density is one and the scaled interaction $\varepsilon^{-2} W((t-s) / \varepsilon)$. We break the systems into blocks of length one. In each block $\sigma$ is constant with a probability approaching one as $\varepsilon \rightarrow 0$. The interaction decays as $1 / t^{2}$ for large $t$. The difficulty arises when trying to estimate the effective nearest neighbor coupling. Taking the worst case, spins a distance two apart, yields $\varepsilon^{-2} W(2 / \varepsilon) \cong 1 / 4$ as $\varepsilon \rightarrow 0$. (This cannot be improved by changing the block size.) The large effective nearest neighbor coupling is due to spins which are close.

Proof of Theorem 2 (iii). (large $\varepsilon$ ) As in [2] we break the continuum spin system in blocks of suitable size. $W(t-s)$ is bounded below by a function constant on each block. We then average for fixed block magnetization and compare to a \pm spin system by means of Wells' inequality.

We set $\alpha_{M F}=\left(\int_{0}^{\infty} d t W(t)\right)^{-1}$ We have to show that if $\tilde{\alpha}>\alpha_{M F}$, then $\langle\sigma(0)\rangle_{+}(\varepsilon \tilde{\alpha}, \varepsilon)>0$ for $\varepsilon$ sufficiently large.

We scale the flip rate into the interaction. Then the flip rate is 1 and the interaction is $\varepsilon^{-2} W((t-s) / \varepsilon)$. The block size is $\varepsilon b$, where $b$ is chosen such that

$$
\tilde{\alpha} \int_{0}^{b} d t W(t)>1
$$

Let

$$
m_{j}=\frac{1}{\varepsilon b} \int_{(j-(1 / 2)) \varepsilon b}^{(j+(1 / 2)) \varepsilon b} d t \sigma(t)
$$

be the magnetization of the $j-t h$ block, $\left|m_{j}\right| \leqq 1$. The lower bound for $\langle\sigma(0)\rangle_{+}(\alpha, \varepsilon)$ is associated with the Hamiltonian

$$
H=-(\varepsilon \tilde{\alpha} / 4) \sum_{i \neq j} b^{2} W(|i-j|+1) m_{i} m_{j}+\text { b.c. }
$$

and the measure 


$$
\frac{1}{Z} \prod_{j} v^{\varepsilon}\left(d m_{j}\right) e^{-H}
$$

The single site measure, $v^{\varepsilon}$, is the distribution of the magnetization $m=1 / \varepsilon b \int_{0}^{\varepsilon b} d t \sigma(t)$ with respect to

$$
\frac{1}{Z} \mu_{[0, \varepsilon b]}^{1}(d \sigma) \exp \left[(\varepsilon \tilde{\alpha} / 4) \int_{0}^{\varepsilon b} d t \int_{0}^{\varepsilon b} d s \varepsilon^{-2} W((t-s) / \varepsilon) \sigma(t) \sigma(s)\right] .
$$

$\mu_{[0, \varepsilon b]}^{1}$ is the spin flip process with rate 1 and free boundary conditions in the interval $[0, \varepsilon b]$.

Equation (6.11) is a standard mean field model. A given spin interacts with the other spins through a weak long-range potential with integrated strength of order one. The mean field model has the critical coupling strength $\tilde{\alpha}_{c}\left(\int_{0}^{b} d t W(t)\right)^{-1}$. Therefore, by our choice of $b$, in the limit $\varepsilon \rightarrow \infty$

$$
v^{\varepsilon}(d m) \rightarrow \frac{1}{2}\left(\delta\left(m-m^{*}\right)+\delta\left(m+m^{*}\right)\right),
$$

where $m^{*}>0$ is the spontaneous magnetization of the mean field model (6.11). $m^{*}$ is independent of $\varepsilon$.

For $\varepsilon$ sufficiently large we can satisfy Wells' inequality with discrete spins of magnitude $m^{*} / 2$. Therefore

$$
\langle\sigma(0)\rangle_{+}(\varepsilon \tilde{\alpha}, \varepsilon) \geqq\left\langle\sigma_{0}\right\rangle_{+}\left(\varepsilon \tilde{\alpha} m^{* 2} / 4\right) .
$$

The left-hand average refers to a discrete Ising model, $\sigma_{j}= \pm 1$, with Hamiltonian

$$
H=-\left(\varepsilon \tilde{\alpha} m^{* 2} / 16\right) \sum_{i \neq j} b^{2} W((|i-j|+1) b) \sigma_{i} \sigma_{j} .
$$

$\tilde{\alpha}, m^{*}$ and $b$ and do not depend on $\varepsilon$. The interaction is $|i-j|^{-2}$ for large separation. Therefore increasing $\varepsilon$ we can achieve that $\left.\left\langle\sigma_{0}\right\rangle_{+}\left(\varepsilon \tilde{\alpha} m^{* 2} / 4\right)\right\rangle 0$.

\section{Jump in the Order Parameter}

For the proof of Theorem 3 we only indicate the major changes as compared to [3, Sect. 4]. This procedure puts a somewhat heavy burden on the reader. The alternative choice - a complete transcription of the proof - would be too much of a repetition.

Since we want to show absence of percolation, the main worry are intervals of points of fracture which are too long. FKG helps again.

Lemma 7.1. Let $n_{f}(\Lambda)$ be the number of points of fracture in the bounded interval A. Then

$$
\mathbf{P}_{2}\left(\left\{n_{f}(\Lambda)=0\right\}\right) \leqq \mathbf{P}_{1}\left(\left\{n_{f}(\Lambda)=0\right\}\right)=e^{-\varepsilon|\Lambda|},
$$

$|\Lambda|$ the length of $\Lambda$.

Proof. $\chi\left(\left\{n_{f}(\Lambda)=0\right\}\right)$ is an increasing function. Thus (7.1) follows from (5.10) 
We note that (7.1) holds also for the measures conditioned on the configuration in an arbitrary subset of $\mathbf{R} \cup \mathbf{R}_{0}^{2}$.

Proof of Theorem 3. (in the spirit of a guiding comment to Sect. 4 of [3]). The strong FKG property holds also for the continuum system, cf. Sect. 5. Let $A$ be the event of specifying all points of fracture and all bonds except in the set $[x, x+d x] \times[y, y+d y]$. Then the conditional probability

$\mathbf{P}_{2}$ (there is a bond from $[x, x+d x]$ to $\left.[y, y+d y] \mid A\right)=\alpha W(y-x) d y d x$.

This identifies $K^{+}(z)=W(z), \alpha=\beta^{+}$. We define

$$
M_{H}=\mathbf{P}_{2, H}(0 \text { is connected to } \mathbf{R} \backslash[-H, H]) \text {. }
$$

$M_{+}=m^{*}$ in our notation. Because of FKG $M_{H}$ is also obtained by conditioning $\mathbf{P}_{2}$ on all fracture points outside $[-H, H]$ and on all bonds with both endpoints in $\mathbf{R} \backslash[-H, H]$ and taking the supremum over the conditioning.

For $x \in \mathbf{R}$ let $C(x)$ be the connected component to which $x$ belongs. $C(x)$ is a countable union of open intervals of the form $\left(\tau_{j-1}, \tau_{j}\right)$. Adapting Definition 4.2 we call a bond $\{x, y\} H$-anchored if $C(x)^{*} \cap(\mathbf{R} \backslash[x-H, x+H]) \neq \emptyset$ and $C(y)^{*} \cap$ $(\mathbf{R} \backslash[y-H, y+H]) \neq \emptyset$, where $C(x)^{*}$ and $C(y)^{*}$ are connected components of $x$ and $y$ obtained when removing the bond $\{x, y\}$.

In Lemma $4.1 A$ is any event determined by bonds with both sites at a distance greater than $H$ from $x$ and $y$ and determined by points of fracture in $\mathbf{R} \backslash([x-H, x+H] \cup[y-H, y+H])$. The estimate reads

$$
\begin{aligned}
& \left.\left.\mathbf{P}_{2} \text { (there is an } H \text { - anchored bond from } x \text { to }[y, y+d y]\right) \mid A\right) \\
& \leqq\left(\beta^{+} M_{H}^{2}+\varepsilon\right) /|x-y|^{2} d y .
\end{aligned}
$$

The modified $A_{L, K}$ and $F_{L, K}$ are given below (6.3). Lemma (4.2) remains unchanged.

$C_{H}(x)=C(x)^{\sim} \cap[x-H, x+H]$, where for the connected component $C(x)^{\sim}$ only bonds with both sites in $[x-H, x+H]$ are admitted. The left anchor is a bond $\left\{w^{\prime}, w\right\}$ such that $w^{\prime} \in C_{H}(x)$ and $w$ is maximal. In Definition 4.3 ii) of a span we add the requirement that the right endpoint is a point of fracture, i.e. the span $[r, s]$ has $r=x$ and $s$ the smallest point of fracture larger than the $s$ of $[3,(4.9)]$.

Lemma 4.3 and 4.4 are unchanged. The bound (4.10) reads now

$$
\mathbf{P}_{2}(-) \leqq \int_{\operatorname{dist}(y, A) \leqq J}\left(\beta^{+} M_{H}^{2}+\varepsilon\right) /|y-x|^{2},
$$

and the bound (4.15) now reads

$$
\mathbf{P}_{2}(-) \leqq 1-\exp \left[-\int_{A} d x \int_{\operatorname{dist}(y, B) \leqq J} d y\left(\beta^{+} M_{H}^{2}+2 \varepsilon\right) /|y-x|^{2}\right] .
$$

In the proof of Lemma 4.3 we have also the case where the distance between $y$ and the smallest point of fracture larger than $y$ is larger than $J$. This case is covered by Lemma 7.1. For (4.12) and (4.13) we use that $W(t)$ is decreasing for $t \geqq 0$.

With the basic estimate (7.6) the remainder of the proof is identical to $[3$, Sec. 4] 


\section{The Boson Density}

Let

$$
n(f)=\int d x f(x) a^{+}(x) a(x)
$$

be the Boson density in position space and let

$$
n_{m}(f)=\int d k f(k) a^{+}(k) a(k)
$$

be the Boson density in momentum space, $f$ some smooth, rapidly decreasing test function. Averaging the Weyl operator, $W(f)$, over the appropriate Gaussian measure yields

$$
\begin{aligned}
\langle\exp [-n(f)]\rangle_{+}= & \left\langle\operatorname { e x p } \left[-\frac{\alpha}{8} \int d t \int d s \sigma(t) \sigma(s)(1-\theta(t) \theta(s))\right.\right. \\
& \left.\left.\cdot \int d k \int d k^{\prime} \lambda\left(k^{\prime}\right) e^{-\omega(k)|t|} e^{-\omega\left(k^{\prime}\right)|s|}\left(1-e^{-f}\right)^{\prime}\left(k-k^{\prime}\right)\right]\right\rangle_{+},
\end{aligned}
$$

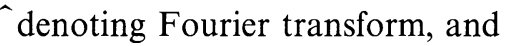

$$
\begin{aligned}
\left\langle\exp \left[-n_{m}(f)\right]\right\rangle_{+}= & \left\langle\operatorname { e x p } \left[-\frac{\alpha}{8} \int d t \int d s \sigma(t) \sigma(s)(1-\theta(t) \theta(s))\right.\right. \\
& \left.\left.\cdot \int d k \lambda(k)^{2} e^{-\omega(k)|t|} e^{-\omega(k)|s|}\left(1-e^{-f}\right)(k)\right]\right\rangle_{+} .
\end{aligned}
$$

We restrict our discussion to $h=0$. If $\alpha \geqq \alpha_{c}$, thus $m^{*}>0$, we substitute $\sigma(t)$ by $\left(\sigma(t)-m^{*}\right)+m^{*}$. In (8.3), (8.4) thereby a term is factored out of the average. This term equals the ground state average of $\exp [-n(f)]$, respectively $\exp \left[-n_{m}(f)\right]$, for the spin-boson Hamiltonian with $\varepsilon=0, h=0$, but an effective coupling constant $\alpha m^{* 2}$.

We also note that the distribution of the number of bosons with momentum in the region $\Lambda$ decays faster than any exponential provided $\int_{\Lambda} d k \lambda(k)^{2} / \omega(k)^{2}<\infty$. If $\int d k e^{i k x} \lambda(k) / w(k)$ is locally $L^{2}$, then the distribution of the number of bosons in any bounded spatial region decays faster than any exponential.

We differentiate (8.3), (8.4) at $f=0$. This yields the average -spatial Boson density

$$
\left\langle a^{+}(x) a(x)\right\rangle_{+}=\frac{\alpha}{2} \int_{0}^{\infty} v(d \lambda)\left|\int d k e^{i k x} \lambda(k) /(\lambda+\omega(k))\right|^{2}
$$

and the average momentum density

$$
\left\langle a^{+}(k) a(k)\right\rangle_{+}=\frac{\alpha}{2} \int_{0}^{\infty} v(d \lambda)[\lambda(k) /(\lambda+\omega(k))]^{2} .
$$

Here $v(d \lambda)$ is the spectral measure of the two-point function of the continuum Ising model,

$$
\langle\sigma(t) \sigma(0)\rangle_{+}=\int_{0}^{\infty} v(d \lambda) e^{-\lambda|t|} .
$$


In particular, the average number, $N$, of bosons in the ground state is

$$
\langle N\rangle=\frac{\alpha}{2} \int_{0}^{\infty} d t t W(t)\langle\sigma(t) \sigma(0)\rangle_{+} .
$$

Thus the average boson density is tied in with the Ising two-point function.

We will prove below that for all $\alpha<\alpha_{c}$,

$$
\langle\sigma(t) \sigma(0)\rangle_{+} \simeq t^{-2}
$$

for large $t$. Close to $\alpha_{c}$, presumably, $\langle\sigma(t) \sigma(0)\rangle_{+} \cong m^{* 2}$ for intermediate $t$ and then it eventually drops as $t^{-2}$ to zero. For $\alpha \geqq \alpha_{c}$ the asymptotic decay of the two-point function is more complicated. (This behavior is proved only for the discrete Ising model [12].) At $\alpha=\alpha_{c}$ the truncated two-point function is expected to decay as

$$
\langle\sigma(t) \sigma(0)\rangle_{+}-m^{* 2} \simeq \frac{1}{\log t} .
$$

For $\alpha>\alpha_{c}$ there is a power law decay as

$$
\langle\sigma(t) \sigma(0)\rangle_{+}-m^{* 2} \simeq t^{-\kappa}
$$

for large $t$. $\kappa$ increases from 0 as $\alpha$ increases beyond $\alpha_{c}$. There exists another critical coupling strength $\overline{\alpha_{c}}$ at which $\kappa=2$ and $\kappa=2$ for $\alpha>\overline{\alpha_{c}} \cdot \alpha_{c} \leqq \alpha<\overline{\alpha_{c}}$ is an intermediate phase. One expects that $\overline{\alpha_{c}}(\varepsilon) \rightarrow 2$ as $\varepsilon \rightarrow 0$ and that $\overline{\alpha_{c}}(\varepsilon)$ has the same slope as $\alpha_{c}(\varepsilon)$ for large $\varepsilon$.

For the sake of discussion let us assume that $\omega(k) \simeq|k|$ for small $k$ and $\omega(k) \geqq$ $\omega_{0}>0$ otherwise. For $\alpha<\alpha_{c}$, we conclude from (8.9) that $v(d \lambda) \simeq \lambda$ for small $\lambda$. Thus

$$
\left\langle a^{+}(x) a(x)\right\rangle_{+} \simeq|x|^{-d-2}
$$

for large $x$ and

$$
\left\langle a^{+}(k) a(k)\right\rangle_{+} \simeq|k|^{-d+2}
$$

for small $k$. At $\alpha_{c}$ the spectral weight at zero, $v(\{0\})$, jumps from zero to $m^{* 2}$. Correspondingly, for $\alpha \geqq \alpha_{c}$ the decay of the Boson density jumps to

$$
\left\langle a^{+}(x) a(x)\right\rangle_{+} \simeq \frac{\alpha}{2} m^{* 2}\left|\int d k e^{i k x} \lambda(k) / \omega(k)\right|^{2} \simeq|x|^{-d}
$$

for large $x$ and to

$$
\left\langle a^{+}(k) a(k)\right\rangle_{+} \simeq \frac{\alpha}{2} m^{* 2} \lambda(k)^{2} \simeq|k|^{-d}
$$

for small $k$.

In particular, we have

$$
\begin{gathered}
\langle N\rangle_{+}=\infty, \text { for } \alpha<\alpha_{c}, \\
N=\infty \text { with probability one, for } \alpha \geqq \alpha_{c} .
\end{gathered}
$$

It remains the task to prove the decay (8.9) for the two-point function. 
Proposition 8.1. Let $h=0$ and $\alpha<\alpha_{c}(\varepsilon)$. Then there exists constants $c_{+}$and $c_{-}$, depending on $\alpha$ and $\varepsilon$, such that

$$
c_{-} /\left(1+t^{2}\right) \leqq\langle\sigma(0) \sigma(t)\rangle_{+} \leqq c_{+} /\left(1+t^{2}\right) .
$$

The lower bound follows from Griffiths inequality. The achievement here is that the upper bound holds all the way to the critical point. As before we follow $[3,4]$. This time the difficulty is that there is no Simon inequality available in the continuum. With a little bit of extra work Lebowitz inequality does equally well for our purposes. Since we work in the one-phase region, we always set $\langle\cdot\rangle=\langle\cdot\rangle_{+}(\alpha, \varepsilon)$. The proof is split up into four lemmas.

Lemma 8.2. Let $0<L<|x|$ and let $\Lambda(L)=\{u, v \mid$ either $|u| \leqq L,|v| \geqq L$ or $|u| \geqq L$, $|v| \leqq L\}$. Then

$$
\begin{aligned}
\langle\sigma(0) \sigma(x)\rangle \leqq & (\alpha / 2) \int d u \int_{\Lambda(L)} d v\langle\sigma(0) \sigma(u)\rangle W(u-v)\langle\sigma(v) \sigma(x)\rangle \\
& +\langle\sigma(0)\rangle_{+, L}\langle\sigma(0) \sigma(|x|-L)\rangle .
\end{aligned}
$$

Proof. For the finite volume $[-T, T]$ the interaction is split as

$$
\begin{aligned}
\int_{[-T, T]^{2}} d t \int d s W_{\lambda}(t, s) \sigma(t) \sigma(s)= & \lambda \int_{\Lambda(L) \wedge[-T, T]^{2}} d t \int d s W(t-s) \sigma(t) \sigma(s) \\
& +\int_{[-T, T]^{2} \backslash \Lambda(L)} d t \int d s W(t-s) \sigma(t) \sigma(s) .
\end{aligned}
$$

We write

$$
\langle\sigma(0) \sigma(x)\rangle_{T}=\langle\sigma(0) \sigma(x)\rangle_{T}^{(L)}+\int_{0}^{1} d \lambda \frac{d}{d \lambda}\langle\sigma(0) \sigma(x)\rangle_{\lambda, T},
$$

where $\langle\cdot\rangle^{(L)}$ refers to the expectation with respect to the equilibrium measure for which the interactions between $[-L, L]$ and $\mathbf{R} \backslash[-L, L]$ are set equal to zero. By GKSII and Lebowitz inequalities we obtain in the limit $T \rightarrow \infty$

$$
\langle\sigma(0) \sigma(x)\rangle \leqq(\alpha / 2) \int d u \int_{\Lambda(L)} d v\langle\sigma(0) \sigma(u)\rangle W(u-v)\langle\sigma(v) \sigma(x)\rangle+\langle\sigma(0) \sigma(x)\rangle^{(L)} .
$$

Let $x>L$. By conditioning on $\sigma(L)$ and $\sigma(-L)$ we obtain

$$
\begin{aligned}
\langle\sigma(0) \sigma(x)\rangle^{(L)}= & \sum_{\sigma, \sigma^{\prime}}\langle\sigma(0) \sigma(x)\rangle_{\sigma(L)=\sigma, \sigma(-L)=\sigma^{\prime}}^{(L)}\left\langle\delta_{\sigma(L), \sigma} \delta_{\sigma(-L), \sigma^{\prime}}\right\rangle^{(L)} \\
= & \sum_{\sigma, \sigma^{\prime}}\langle\sigma(0)\rangle_{\sigma(L)=\sigma, \sigma(-L)=\sigma^{\prime}}^{(L)}\langle\sigma(x)\rangle_{\sigma(L)=\sigma, \sigma(-L)=\sigma^{\prime}}^{(L)}\left\langle\delta_{\sigma(L), \sigma} \delta_{\sigma(-L), \sigma^{\prime}}\right\rangle^{(L)} \\
= & \langle\sigma(0)\rangle_{\sigma(L)=1, \sigma(-L)=1}^{(L)}\left\{\langle\sigma(x)\rangle_{\sigma(L)=1, \sigma(-L)=1}^{(L)}\right. \\
& \left.-\langle\sigma(x)\rangle_{\sigma(L)=-1, \sigma(-L)=-1}^{(L)}\right\}\left\langle\delta_{\sigma(L), 1} \delta_{\sigma(-L), 1}\right\rangle^{(L)}
\end{aligned}
$$

where we used the Markov property of the free measure and symmetry. In the expression inside the curly brackets we cut out the interval $[-L, L]$ and join the resulting two pieces together. This gives the correlation $2\langle\sigma(L) \sigma(x)\rangle_{\sim}$ with an 
interaction smaller than $W$. The first factor is bounded by $\langle\sigma(0)\rangle_{+, L}$ and the third factor is bounded by $\frac{1}{2}$.

To shorten our notation we set $\tau(x)=\langle\sigma(0) \sigma(x)\rangle .0 \leqq \tau(x) \leqq 1, \tau(0)=1, \tau(x)=$ $\tau(-x)$ and $\tau(x)$ is monotone decreasing for $x \geqq 0$.

Lemma 7.3. Let

$$
\gamma_{L}=\int d u \int_{\Lambda(L)} d v \tau(u) W(u-v)
$$

The two-point function satisfies the weak bound

$$
\tau(x) \leqq \text { const } \exp \left[-(\log (1+|x|))^{1 / 2}\right]
$$

implying that

$$
\lim _{L \rightarrow \infty} \gamma_{L}=0 .
$$

Proof. The bound (8.25) is a consequence of the percolation analysis [3], compare with Sect. 6.

We choose $x_{0}>L$ such that

$$
W(u) \leqq c / u^{2}
$$

for $|u|>x_{0}$. We partition $\Lambda(L) \cap\{u+v \geqq 0\}$ into four pieces. The remainder, $\Lambda(L) \cap\{u+v \leqq 0\}$, follows by symmetry.

(1) $L-x_{0} \leqq u \leqq L, L \leqq v$. The integral is bounded by $\int d v W(v) \int_{-x_{0}}^{L} d u \tau(u)$ which tends to zero as $L \rightarrow \infty$.

(2) $-L \leqq u \leqq L-x_{0}, L \leqq v$. This case is discussed in [3].

(3) $L \leqq u \leqq L+x_{0},-L \leqq v \leqq L$. The integral is bounded by $\int d v W(v) \int_{L}^{L+x_{0}} d u \tau(u)$ which tends to zero as $L \rightarrow \infty$.

(4) $L+x_{0} \leqq u,-L \leqq v \leqq L$. The. integral is bounded by

$$
c \int_{L+x_{0}}^{\infty} d u \int_{-L}^{L} d v \tau(u)(u-v)^{-2} \leqq c^{\prime}(\log L) \tau(L)
$$

which tends to zero as $L \rightarrow \infty$

Lemma 8.4. The susceptibility is bounded,

$$
\int d x \tau(x)<\infty .
$$

Proof. We integrate Lebowitz inequality (8.19). Then for $M>L$

$$
\begin{aligned}
\int_{-M}^{M} d x \tau(x) \leqq & \int_{-L}^{L} d x \tau(x)+\int_{\Lambda(L)} d u \int d v \tau(u) W(u-v) \sup _{v^{\prime}} \int_{-M}^{M} d \tau \tau\left(v^{\prime}-x\right) \\
& +\langle\sigma(0)\rangle_{+, L} \int_{|x| \leq M-L} d x \tau(x),
\end{aligned}
$$


and therefore

$$
\left[1-\gamma_{L}-\langle\sigma(0)\rangle_{+, L}\right] \int_{-M}^{M} d x \tau(x) \leqq \int_{-L}^{L} d x \tau(x) .
$$

$L$ sufficiently large and $M \rightarrow \infty$ yields a finite susceptibility

Lemma 7.5. There exists some $\hat{L}$ such that for all $x>\hat{L}$

$$
\tau(x) \leqq c / x^{2}+\beta \tau(x / 2)
$$

with $0<\beta<\frac{1}{4}$.

Proof. For each $x>3 x_{0}$ we apply (8.19) with $\Lambda(x / 6)$,

$$
\tau(x) \leqq \int_{\Lambda(x / 6)} d u \int d v \tau(u) W(u-v) \tau(v-x)+\langle\sigma(0)\rangle_{+, x / 6} \tau(5 x / 6) .
$$

As before, we partition the domain of integration. If either $|u| \leqq x / 6,|v| \geqq x / 2$ or $|u| \geqq x / 2,|v| \leqq x / 6$, then the supremum of $W$ is bounded by const $/ x^{2}$ and $\int d u \tau(u)$ is bounded according to Lemma 7.4. On the other hand if either $|u| \leqq x / 6, x / 6 \leqq|v|$ $\leqq x / 2$ or $x / 6 \leqq|u| \leqq x / 2,|v| \leqq x / 6$, then the integral is bounded by $\gamma(x / 6) \tau(x / 2)$. Therefore

$$
\tau(x) \leqq c / x^{2}+\left(\left(\gamma_{x} / 6\right)+\langle\sigma(0)\rangle_{+, x / 6}\right) \tau(x / 12)
$$

for all $x>3 x_{0}$. Since $\gamma_{x / 6}$ and $\langle\sigma(0)\rangle_{+, x / 6}$ tend to zero as $x \rightarrow \infty,(8.32)$ follows

The proof of Proposition 8.1 follows by iterating (8.32), cf. [3].

Acknowledgements. It is a pleasure to thank J. Bricmont, J. Chayes, L. Chayes and C. Newman for informative discussions. I am grateful to J. Cardy for his kind hospitality at the Department of Physics.

\section{References}

1. Leggett, A. J., Chakravarty, S., Dorsey, A. T., Fisher, M. P. A., Garg, A., Zwerger, W.: Dynamics of the dissipative two-state system. Rev. Mod. Phys 59, 1 (1987)

2. Spohn, H., Dümcke, R.: Quantum tunneling with dissipation and the Ising model over R. J. Stat. Phys. 41, 389 (1985)

3. Aizenman, M., Newman, C. M.: Discontinuity of the percolation density in one dimensional $1 /|x-y|^{2}$ percolation models. Commun. Math. Phys. 107, 611 (1986)

4. Aizenman, M., Chayes, J. T., Chayes, L., Newman, C. M.: Discontinuity of the magnetization in one dimensional $1 /|x-y|^{2}$ Ising and Potts models. J. Stat. Phys. 50, 1 (1988)

5. Fannes, M., Nachtergaele, B., Verbeure, A.: The equilibrium states of the spin-boson model. Commun. Math. Phys. 114, 537 (1988)

6. Bratteli, O., Robinson, D. W.: Operator algebras and quantum statistical mechanics, vol. II. Berlin, Heidelberg, New York: Springer 1981

7. Ginibre, J.: Some applications of functional integration in statistical mechanics. In: Statistical mechanics and field theory. DeWitt, C., Stora, R. (eds.). New York: Gordon and Breach 1971

8. Lebowitz, J. L., Martin-Löf, A.: On the uniqueness of the equilibrium state for Ising spin systems. Commun. Math. Phys. 25, 276 (1972)

9. Lebowitz, J. L.: Coexistence of phases in Ising ferromagnets. J. Stat. Phys. 16, 463 (1977)

10. Bricmont J., Lebowitz, J. L.: On the continuity of the magnetization and energy in Ising ferromagnets. J. Stat. Phys. 42, 861 (1986)

11. Newman, C. M., Schulman, L. S.: One dimensional $1 /|i-j|^{s}$ percolation models: The existence of a transition for $s \leqq 2$. Commun. Math. Phys. 104, 547 (1986) 
12. Imbrie, J. Z., Newman, C. M.: An intermediate phase with slow decay of correlations in one dimensional $1 /|x-y|^{2}$ percolation, Ising and Potts models. Commun Math. Phys. 118, 303 (1988)

13. Thouless, J. D.: Long range order in one-dimensional Ising systems. Phys. Rev. 187, 732 (1969)

14. Fröhlich, J., Isreal, R. B., Lieb, E. H., Simon, B.: Phase transitions and reflection positivity, I. General theory and long-range lattice models. Commun. Math. Phys. 62, 1-34 (1980)

Communicated by M. Aizenman

Received July 1, 1988; in revised form November 4, 1988 